\title{
On the interior motive of certain Shimura varieties: the case of Hilbert-Blumenthal varieties
}

\author{
by \\ Jörg Wildeshaus * \\ LAGA \\ UMR 7539 \\ Institut Galilée \\ Université Paris 13 \\ Avenue Jean-Baptiste Clément \\ F-93430 Villetaneuse \\ France \\ wildesh@math.univ-paris13.fr
}

March 18, 2011

\begin{abstract}
The purpose of this article is to construct a Hecke-equivariant Chow motive whose realizations equal interior (or intersection) cohomology of Hilbert-Blumenthal varieties with non-constant algebraic coefficients.
\end{abstract}

Keywords: Hilbert-Blumenthal varieties, weight structures, boundary motive, interior motive.

Math. Subj. Class. (2000) numbers: 14G35 (11F32, 11F41, 14C25, 14F25, 19E15, 19F27)

${ }^{*}$ Partially supported by the Agence Nationale de la Recherche, project no. ANR-07BLAN-0142 "Méthodes à la Voevodsky, motifs mixtes et Géométrie d'Arakelov". 


\section{Contents}

1 Introduction $\quad 2$

2 A criterion on the existence of the interior motive 7

3 Statement of the main results 11

4 Proof of the main results

\section{Introduction}

The purpose of this paper is the construction and the analysis of the interior motive of Kuga-Sato families over Hilbert-Blumenthal varieties associated to a fixed totally real number field $L$.

In order to motivate the problem, let us start by discussing the case of classical modular curves, i.e., of Hilbert-Blumenthal varieties associated to $L=\mathbb{Q}$. Fix two integers $n \geq 3$ and $r \geq 1$, let $S$ denote the modular curve parametrizing elliptic curves with level $n$ structure, and $S \hookrightarrow \bar{S}$ its smooth compactification. Write $A \rightarrow S$ for the universal elliptic curve, and $\bar{A} \rightarrow \bar{S}$ for the universal generalized elliptic curve. Thus, $\bar{A}$ is smooth and proper over $\mathbb{Q}$. The $r$-fold fibre product $\bar{A}^{r}:=\bar{A} \times_{\bar{S}} \times \ldots \times_{\bar{S}} \bar{A}$ of $\bar{A}$ over $\bar{S}$ is singular for $r \geq 2$, and can be desingularized canonically [D]. Denote by $\overline{\bar{A}}^{r}$ this desingularization. The symmetric group $\mathfrak{S}_{r}$ acts on $\bar{A}$ by permutations, the $r$-th power of the group $\mathbb{Z} / n \mathbb{Z}$ by translations, and the $r$-th power of the group $\mu_{2}$ by inversion in the fibres. Altogether, this gives a canonical action of the semi-direct product

$$
\Gamma_{r}:=\left((\mathbb{Z} / n \mathbb{Z})^{2} \rtimes \mu_{2}\right)^{r} \rtimes \mathfrak{S}_{r}
$$

by automorphisms on $\bar{A}^{r}$. By the canonical nature of the desingularisation, this extends to an action of $\Gamma_{r}$ by automorphisms on $\overline{\bar{A}}^{r}$.

Let $\varepsilon: \Gamma_{r} \rightarrow\{ \pm 1\}$ be the morphism which is trivial on $(\mathbb{Z} / n \mathbb{Z})^{2 r}$, is the product map on $\mu_{2}^{r}$, and is the sign character on $\mathfrak{S}_{r}$. Let $e$ denote the idempotent in the group ring $\mathbb{Q}\left[\Gamma_{r}\right]$ associated to $\varepsilon$. Following [Sch], one defines the Chow motive ${ }_{n}^{r} \mathcal{W}$ as the image

$$
{ }_{n}^{r} \mathcal{W}:=M_{g m}\left(\overline{\bar{A}}^{r}\right)^{e}
$$

of the idempotent $e$ on the Chow motive $M_{g m}\left(\overline{\bar{A}}^{r}\right)$ of the smooth and proper Q-scheme $\overline{\bar{A}}^{r}$. 
By [Sch], the (Betti or $\ell$-adic) realizations of ${ }_{n}^{r} \mathcal{W}$ equal interior cohomology, i.e., the image of the morphism

$$
H_{c}^{n}\left(A^{r}\right)^{e} \longrightarrow H^{n}\left(A^{r}\right)^{e}
$$

linking (Betti or $\ell$-adic) cohomology with compact support to cohomology without support of the $r$-fold fibre product $A^{r}$ of $A$ over $S$. Note that at places $p$ of good reduction of $\overline{\bar{A}}^{r}$ (i.e., $p$ does not divide $n$ ), this result implies reduction properties for the Galois action on $\ell$-adic interior cohomology [Sch, Thm. 1.2.4].

Using the Eichler-Shimura isomorphism, the Hodge structure or Galois module associated to an elliptic normalized newform $f$ of level $n$ and weight $r+2$ can be realized in (the image of $e$ on) interior cohomology of $A^{r}$. This realization coincides with the eigenspace of the action of the Hecke algebra associated to the eigenvalues $a_{p}$ occurring in the Fourier expansion of $f$ [D]. In order to lift this construction to motives, one first extends the Hecke correspondences to $\overline{\bar{A}}^{r}$, and checks that they commute with the action of $\Gamma_{r}$ [Sch, Sect. 4.1]. Scholl then constructs the Grothendieck motive $M(f)$ associated to $f$ as the factor of the Grothendieck motive underlying the Chow motive ${ }_{n}^{r} \mathcal{W}$ corresponding to the same eigenvalues $a_{p}$ [Sch, Sect. 4.2].

Let us agree on the principle that in order to geometrically explain certain purity phenomena in Hodge or Galois theory, it is desirable to construct (Chow or Grothendieck) motives whose realizations equal the Hodge structure or Galois module in question.

In the setting discussed above, purity concerns interior cohomology of the smooth non-proper scheme $A^{r}$, and more specifically, the weight occurring in the Hodge structure or Galois module associated to the modular form $f$. The motives ${ }_{n}^{r} \mathcal{W}$ and $M(f)$ are constructed out of a smooth and proper scheme. Given the definition of interior cohomology, it is natural to look for this scheme among the smooth compactifications of $A^{r}$.

Let us insist on one decisive additional point: among the smooth compactifications of $A^{r}$, there is one choice (namely $\overline{\bar{A}}^{r}$ ) allowing for extensions of the actions on $A^{r}$ of both the finite group $\Gamma_{r}$ and the Hecke correspondences. Unfortunately, this additional point is rather atypical: smooth equivariant compactifications are not known (and maybe not reasonable to expect) to exist for Shimura varieties of higher dimensions. Thus, it appears unrealistic to hope for a naïve generalization of Scholl's approach to such varieties.

In the present paper, we shall develop an alternative strategy, based on the formalism of weight structures [Bo]. More precisely, we shall use the motivic weight structure of [loc. cit.], with which the category $D M_{g m}^{e f f}(k)$ of 
effective geometrical motives over a perfect field $k[\mathrm{~V}]$ is equipped.

Since our strategy gives the same result as Sch for modular curves, let us continue to discuss that case, and make the necessary translations. For a smooth scheme over $k$, denote by $M_{g m}(X)$ and $M_{a m}^{c}(X)$ the motive of $X$ and its motive with compact support, respectively $\mathrm{V}$. Effective Chow motives form a full sub-category of $D M_{g m}^{e f f}(k)$; indeed they are identified with the category of objects which are pure of weight zero with respect to the motivic weight structure [Bo]. Both functors $M_{g m}$ and $M_{g m}^{c}$ agree on smooth and proper $k$-schemes $X$, and yield the Chow motive of $X$. In particular, we may consider ${ }_{n}^{r} \mathcal{W}$ as a geometrical motive over $\mathbb{Q}$. Thanks to the functoriality properties of $M_{g m}$ and $M_{g m}^{c}$, we get natural morphisms

$$
j^{r, *}:{ }_{n}^{r} \mathcal{W}=M_{g m}\left(\overline{\bar{A}}^{r}\right)^{e} \longrightarrow M_{g m}^{c}\left(A^{r}\right)^{e}
$$

and

$$
j^{r}: M_{g m}\left(A^{r}\right)^{e} \longrightarrow M_{g m}\left(\overline{\bar{A}}^{r}\right)^{e}={ }_{n}^{r} \mathcal{W}
$$

induced by the open immersion $j^{r}$ of $A^{r}$ into $\overline{\bar{A}}^{r}$. The analysis from Sch, Sect. 2 and 3] of the geometric properties of the boundary of $\overline{\bar{A}}^{r}$ can then be employed to prove [W4, Sect. 3] that $j^{r, *}$ and $j^{r}$ factor canonically through isomorphisms

$$
{ }_{n}^{r} \mathcal{W} \stackrel{\sim}{\longrightarrow} \operatorname{Gr}_{0} M_{g m}^{c}\left(A^{r}\right)^{e}
$$

and

$$
\mathrm{Gr}_{0} M_{g m}\left(A^{r}\right)^{e} \stackrel{\sim}{\longrightarrow} \mathcal{W}
$$

Here, the symbol $\mathrm{Gr}_{0}$ denotes the "graded part of weight zero" with respect to the motivic weight structure. It is defined on motives enjoying an additional property, which therefore turns out to be essential for the present paper: the property of avoiding weights -1 and 1 . Both $M_{g m}^{c}\left(A^{r}\right)^{e}$ and $M_{g m}\left(A^{r}\right)^{e}$ satisfy this hypothesis, thanks again to the analysis of the boundary of $\overline{\bar{A}}^{r}$ [Sch].

To resume, we see that the canonical morphism $M_{g m}\left(A^{r}\right)^{e} \rightarrow M_{g m}^{c}\left(A^{r}\right)^{e}$ factors canonically through an isomorphism

$$
\operatorname{Gr}_{0} M_{g m}\left(A^{r}\right)^{e} \stackrel{\sim}{\longrightarrow} \operatorname{Gr}_{0} M_{g m}^{c}\left(A^{r}\right)^{e} .
$$

Our key observation is that this latter statement does no longer necessitate the reference to a compactification of $A^{r}$ ! In fact, it is this statement that turns out to generalize, with appropriate choices of $e$ (see below) to Hilbert-Blumenthal varieties of arbitrary dimension. Since it concerns the "graded part of weight zero" of the canonical morphism relating $M_{g m}\left(A^{r}\right)^{e}$ to $M_{g m}^{c}\left(A^{r}\right)^{e}$, it necessitates control of the weights present in a cone of that morphism. 
Let us make this a little more precise. Recall that for a smooth scheme $X$ over $k$, the boundary motive $\partial M_{g m}(X)$ of $X[\mathrm{~W} 2$ is a canonical choice of (the shift by [-1] of) such a cone; indeed, it fits into a canonical exact triangle

$$
(*) \quad \partial M_{g m}(X) \longrightarrow M_{g m}(X) \longrightarrow M_{g m}^{c}(X) \longrightarrow \partial M_{g m}(X)[1] .
$$

Given the specific choice of cone, the boundary motive satisfies good functorial properties. Assume that an idempotent endomorphism $e$ of the exact triangle $(*)$ is given. We thus get a direct factor

$$
\partial M_{g m}(X)^{e} \longrightarrow M_{g m}(X)^{e} \longrightarrow M_{g m}^{c}(X)^{e} \longrightarrow \partial M_{g m}(X)^{e}[1] \text {. }
$$

In view of the above, let us assume the object $\partial M_{g m}(X)^{e}$ to avoid weights -1 and 0 with respect fo the motivic weight structure. As verified in [W4, this assumption is not only necessary, but also sufficient to construct the Chow motives $\operatorname{Gr}_{0} M_{g m}(X)^{e}$ and $\operatorname{Gr}_{0} M_{g m}^{c}(X)^{e}$, and to show that they are canonically isomorphic. Given the nature of the realizations of $\operatorname{Gr}_{0} M_{g m}(X)^{e}$, it is natural to call this object the $e$-part of the interior motive of $X$.

In the context of Hilbert-Blumenthal varieties, it thus remains to verify the hypothesis on the absence of weights -1 and 0 in $\partial M_{g m}(X)^{e}$, and technically speaking, this is what the present paper is about. In Section 2, we establish a criterion (Theorem 2.2) allowing for that verification, when the boundary motive is Artin-Tate. Its simplified form (Corollary 2.4) states that the absence of weights can be read off from the Hodge structure or the Galois action on boundary cohomology.

Section 3 contains the statements of our main results, Theorems 3.5 and 3.6. Here, $X$ is the $r$-th power of the universal Abelian scheme over a smooth Hilbert-Blumenthal variety $S$ associated to a totally real number field $L$ of degree $g$. The idempotent $e$ cuts out the direct factor of the relative Chow motive of $X$, on which the action of $L$ is of type $\left(r_{1}, \ldots, r_{g}\right)$, for $r_{1}+\ldots+r_{g}=r$. Theorem 3.5 implies in particular that in this context, the criterion from Theorem 2.2 is satisfied as soon as $r \geq 1$. Therefore, the interior motive $\mathrm{Gr}_{0} M_{g m}(X)^{e}$ exists. We list its principal properties, using the main results from [W4, Sect. 4]. First (Corollary [3.7), we get precise statements on the weights occurring in the motive $M_{g m}(X)^{e}$ and the motive with compact support $M_{g m}^{c}(X)^{e}$. Second (Corollary 3.8), the interior motive is Hecke-equivariant; it is here that the functorial properties of the boundary motive turn out to be essential. Corollary 3.8 appears particularly interesting, given the problem of non-existence of equivariant smooth compactifications of $X$ (for $g \geq 2$ ) raised above. Third (Corollary [3.9), the interior motive occurs canonically as a direct factor of the (Chow) motive of any smooth compactification of $X$. Let us mention that in the case of "nonparallel type", i.e., the integers $r_{i}$ used to construct the idempotent $e$ are not all equal to each other, Theorem 3.6 states that the $e$-part of the boundary motive vanishes. In particular, the interior motive then coincides with the 
e-part of the motive of the (open) Kuga-Sato variety. This can be seen as a motivic explanation of [F, Rem. I.4.8]: "If $f$ is a modular form, but not a cusp form, then $r_{1}=\ldots=r_{g}$." We then discuss the special cases $g=1$ and $g=2$. For $g=2$, the control of the weights from Corollary 3.7 turns out to be sufficiently precise to allow for a strenghtening of the main result of [K] (Corollaries 3.13 and 3.14): the special elements in the motivic cohomology of $X$ constructed in [loc. cit.] come indeed from motivic cohomology of a (in fact, of any) smooth compactification of $X$.

The final Section 4 is devoted to the verification of the criterion from Corollary 2.4 for Hilbert-Blumenthal varieties. First, we need to show that in this case, the boundary motive is indeed Artin-Tate (Theorem 4.2). This is done using a smooth toroidal compactification. We use co-localization for the boundary motive [W2], in order to reduce to showing the statement for the contribution of any of the strata. The latter was identified in the general context of mixed Shimura varieties [W3]. For Kuga-Sato families over Hilbert-Blumenthal varieties, [loc. cit.] shows in particular that these contributions are indeed all Artin-Tate. We are thus reduced to the identification of boundary cohomology. The resulting formula is most certainly known to the experts (see e.g. [BrL, $\mathrm{Ha}, \overline{\mathrm{Bl}}]$ ). In the presence of a "non-parallel type", it actually implies the vanishing of boundary cohomology. In the remaining case, we employ the main result from [BuW], to identify the weights, thereby completing the verification of the criterion from Corollary 2.4.

We should warn the reader that our constructions work a priori with $\mathbb{Q}$-coefficients. This seems to be necessary for at least the following reasons. First, the results on Artin-Tate motives from Section 2 are not known to hold before passage to $\mathbb{Q}$-coefficients; actually, it is not even clear how to formulate the integral version of these results. Second, the construction of the idempotent $e$ relies on the motivic decomposition of Abelian schemes of relative dimension greater than one [DeM]. But this decomposition necessitates the inversion of at least one prime, and is only known to be canonical after $\otimes \mathbb{Q}$. Finally, our computations of the boundary cohomology of HilbertBlumenthal varieties (see Section 4) are valid only after tensoring with $\mathbb{Q}$. In fact, unless one deals with modular curves, very little seems to be known about the primes dividing the torsion of boundary cohomology with integer coefficents (see [Gh, Sect. 3.4]).

Part of this work was done while I was enjoying a modulation de service pour les porteurs de projets de recherche, granted by the Université Paris 13. I wish to thank D. Blasius, M. Dimitrov, V. Maillot, R. Pink and J. Tilouine for useful discussions and comments.

Notation and conventions: Throughout the article, $k$ denotes a fixed number field. We denote by $S c h / k$ the category of separated schemes of finite 
type over $k$, and by $S m / k \subset S c h / k$ the full sub-category of objects which are smooth over $k$. As far as motives are concerned, the notation of this paper is that of [W2, W3, W4, which in turn follows that of [V]. We refer to [W2, Sect. 1] for a concise review of this notation, and of the definition of the triangulated categories $D M_{g m}^{e f f}(k)$ and $D M_{g m}(k)$ of (effective) geometrical motives over $k$. Let $F$ be a commutative $\mathbb{Q}$-algebra. The notation $D M_{g m}^{e f f}(k)_{F}$ and $D M_{g m}(k)_{F}$ stands for the $F$-linear analogues of these triangulated categories defined in An, Sect. 16.2.4 and Sect. 17.1.3]. Similarly, let us denote by $C H M^{e f f}(k)$ and $C H M(k)$ the categories opposite to the categories of (effective) Chow motives, and by $C H M^{\text {eff }}(k)_{F}$ and $C H M(k)_{F}$ the pseudoAbelian completion of the category $C H M^{\text {eff }}(k) \otimes_{\mathbb{Z}} F$ and $C H M(k) \otimes_{\mathbb{Z}} F$, respectively. Using [V], Cor. 4.2.6], we canonically identify $C H M^{e f f}(k)_{F}$ and $C H M(k)_{F}$ with a full additive sub-category of $D M_{g m}^{e f f}(k)_{F}$ and $D M_{g m}(k)_{F}$, respectively.

\section{A criterion on the existence of the interior motive}

Fix $X \in S m / k$, and consider the exact triangle

$(*) \quad \partial M_{g m}(X) \longrightarrow M_{g m}(X) \longrightarrow M_{g m}^{c}(X) \longrightarrow \partial M_{g m}(X)[1]$

in $D M_{g m}^{e f f}(k)$. Recall from [W4, Def. $4.1(\mathrm{a})$ ] that $c(X, X)$ contains a canonical sub-algebra $c_{1,2}(X, X)$ (of "bi-finite correspondences") acting on $(*)$. Denote by $\bar{c}_{1,2}(X, X)$ the quotient of $c_{1,2}(X, X)$ by the kernel of this action. Fix a finite direct product $F$ of fields of characteristic zero, and an idempotent $e$ in $\bar{c}_{1,2}(X, X) \otimes_{\mathbb{Z}} F$. Denote by $M_{g m}(X)^{e}, M_{g m}^{c}(X)^{e}$ and $\partial M_{g m}(X)^{e}$ the images of $e$ on $M_{g m}(X), M_{g m}^{c}(X)$ and $\partial M_{g m}(X)$, respectively, considered as objects of the category $D M_{g m}^{e f f}(k)_{F}$. Recall the following assumption.

Assumption 2.1 ([W4, Asp. 4.2]). The object $\partial M_{g m}(X)^{e}$ is without weights -1 and 0 .

Here, absence of certain weights is in the sense of [W4, Def. 1.10]. In order to apply the results from [W4, Sect. 4], allowing in particular to construct the interior motive, one needs to verify Assumption 2.1. For this purpose, let us consider realizations ([Hu, Sect. 2.3 and Corrigendum]; see [DG, Sect. 1.5] for a simplification of this approach). We shall concentrate on two realizations (the statement from Theorem 2.2 below then formally generalizes to any of the other realizations "with weights" considered in $[\mathrm{Hu}])$ :

(i) the Hodge theoretic realization

$$
R_{\sigma}: D M_{g m}(k)_{F} \longrightarrow D
$$


associated to a fixed embedding $\sigma$ of the number field $k$ into the field $\mathbb{C}$ of complex numbers. Here, $D$ is the bounded derived category of mixed graded-polarizable $\mathbb{Q}$-Hodge structures [Be2, Def. 3.9, Lemma 3.11], tensored with $F$,

(ii) the $\ell$-adic realization

$$
R_{\ell}: D M_{g m}(k)_{F} \longrightarrow D
$$

for a prime $\ell$. Here, $D$ is the bounded "derived category" of constructible $\mathbb{Q}_{\ell}$-sheaves on $\operatorname{Spec}(k)$ [E, Sect. 6], tensored with $F$.

Choose and fix one of these two, denote it by $R$, and recall that it is a contravariant tensor functor mapping the pure Tate motive $\mathbb{Z}(m)$ to the pure Hodge structure $\mathbb{Q}(-m)$ (when $R=R_{\sigma}$ ) and to the pure $\mathbb{Q}_{\ell}$-sheaf $\mathbb{Q}_{\ell}(-m)$ (when $R=R_{\ell}$ ), respectively [Hu, Thm. 2.3.3]. The category $D$ is equipped with a $t$-structure; write $H^{n}$ for the cohomology functors.

Theorem 2.2. Let $\alpha \leq \beta$ be two integers, and $R$ one of the two realizations considered above (Hodge theoretic or $\ell$-adic). Assume that $\partial M_{g m}(X)^{e}$ is a successive extension of objects $M$ of $D M_{g m}^{e f f}(k)_{F}$, each satisfying one of the following properties.

(i) $M$ is without weights $\alpha, \ldots, \beta$.

(ii) $M$ lies in the triangulated sub-category $D M A T(k)_{F}$ of $D M_{g m}(k)_{F}$ of Artin-Tate motives over $k$ [W5, Def. 1.3], and the cohomology object $H^{n} R(M)$ of its image $R(M)$ under $R$ is without weights $n-\beta, \ldots, n-\alpha$, for all $n \in \mathbb{Z}$.

Then $\partial M_{g m}(X)^{e}$ is without weights $\alpha, \ldots, \beta$.

Proof. $\quad$ Apply [W5, Prop. 2.11, Thm. 3.11 (d)]. q.e.d.

Corollary 2.3. If the hypotheses of Theorem 2.2 are met with $\alpha \leq-1$ and $\beta \geq 0$, then Assumption 2.1 holds.

As far as the remaining part of this article is concerned, we shall be dealing with a situation in which the whole of $\partial M_{g m}(X)^{e}$ satisfies property (ii) from Theorem 2.2. It will be worthwile to spell out that property.

Corollary 2.4. The conclusion of Theorem 2.2 holds in particular if $\partial M_{g m}(X)^{e}$ lies in DMAT $(k)_{F}$, and if the e-part of the boundary cohomology of $X$

(in the Hodge theoretic setting) resp.

$$
\left(\partial H^{n}(X(\mathbb{C}), \mathbb{Q}) \otimes_{\mathbb{Q}} F\right)^{e}
$$

$$
\left(\partial H^{n}\left(X_{\bar{k}}, \mathbb{Q}_{\ell}\right) \otimes_{\mathbb{Q}} F\right)^{e}
$$


(in the $\ell$-adic setting) is without weights $n-\beta, \ldots, n-\alpha$, for all $n \in \mathbb{Z}$. If this latter condition is fulfilled with $\alpha \leq-1$ and $\beta \geq 0$, then Assumption 2.1 holds.

Recall that boundary cohomology of $X$ is defined via a compactification $j: X \hookrightarrow \bar{X}$ : writing $i: \partial \bar{X} \hookrightarrow \bar{X}$ for the complementary immersion, one defines $\partial H^{n}(\bullet)$ as cohomology of $\partial \bar{X}$ with coefficients in $i^{*} R j_{*}(\bullet)$. Thanks to proper base change, this definition is independent of the choice of $j$, as is the long exact cohomology sequence

$$
\begin{aligned}
& \ldots \longrightarrow H^{n}(X(\mathbb{C}), \mathbb{Q}) \otimes_{\mathbb{Q}} F \longrightarrow \partial H^{n}(X(\mathbb{C}), \mathbb{Q}) \otimes_{\mathbb{Q}} F \longrightarrow \\
& H_{c}^{n+1}(X(\mathbb{C}), \mathbb{Q}) \otimes_{\mathbb{Q}} F \longrightarrow H^{n+1}(X(\mathbb{C}), \mathbb{Q}) \otimes_{\mathbb{Q}} F \longrightarrow \ldots
\end{aligned}
$$

(in the Hodge theoretic setting) resp.

$$
\begin{aligned}
& \ldots \longrightarrow H^{n}\left(X_{\bar{k}}, \mathbb{Q}_{\ell}\right) \otimes_{\mathbb{Q}} F \longrightarrow \partial H^{n}\left(X_{\bar{k}}, \mathbb{Q}_{\ell}\right) \otimes_{\mathbb{Q}} F \longrightarrow \\
& H_{c}^{n+1}\left(X_{\bar{k}}, \mathbb{Q}_{\ell}\right) \otimes_{\mathbb{Q}} F \longrightarrow H^{n+1}\left(X_{\bar{k}}, \mathbb{Q}_{\ell}\right) \otimes_{\mathbb{Q}} F \longrightarrow \ldots
\end{aligned}
$$

(in the $\ell$-adic setting).

Note also that the algebra $\bar{c}_{1,2}(X, X)$ acts contravariantly on the boundary cohomology $\partial H^{n}(X(\mathbb{C}), \mathbb{Q})$ resp. $\partial H^{n}\left(X_{\bar{k}}, \mathbb{Q}_{\ell}\right)$.

Corollary 2.4 results from Theorem 2.2 and the following.

Proposition 2.5. Fix $X \in S m / k$, a $\mathbb{Q}$-algebra $F$, and e as before. Then $H^{n} R\left(\partial M_{g m}(X)^{e}\right)$ is isomorphic to $\left(\partial H^{n}(X(\mathbb{C}), \mathbb{Q}) \otimes_{\mathbb{Q}} F\right)^{e}$ (in the Hodge theoretic setting) resp. $\left(\partial H^{n}\left(X_{\bar{k}}, \mathbb{Q}_{\ell}\right) \otimes_{\mathbb{Q}} F\right)^{e}$ (in the $\ell$-adic setting), for all $n$.

Proof. It suffices to show that the image under $R$ of the canonical morphism

$$
\iota: M_{g m}(X) \longrightarrow M_{g m}^{c}(X)
$$

can be $c_{1,2}(X, X)$-equivariantly identified with the canonical morphism

$$
R \Gamma_{c}(X) \longrightarrow R \Gamma(X)
$$

in the target $D$ of $R$ of classes of complexes $R \Gamma_{c}(X)$ and $R \Gamma(X)$ computing cohomology with resp. without support. Indeed, the exact triangle $(*)$ will then show that the e-part $\partial M_{g m}(X)^{e}$ of the boundary motive is mapped to a cone of $R \Gamma_{c}(X)^{e} \rightarrow R \Gamma(X)^{e}$. It will therefore be isomorphic to the class of a complex computing the $e$-part of boundary cohomology.

We may assume that $X$ is of pure dimension $d$. First note that for any fixed smooth compactification $j: X \hookrightarrow \bar{X}$, the morphism $\iota$ is the composition of the canonical morphism

$$
M_{g m}(j): M_{g m}(X) \longrightarrow M_{g m}(\bar{X}),
$$


of the inverse of the duality isomorphism,

$$
M_{g m}(\bar{X}) \stackrel{\sim}{\longrightarrow} M_{g m}(\bar{X})^{*}(d)[2 d]
$$

[V, Thm. 4.3.7 3], of the dual of $M_{g m}(j)$,

$$
M_{g m}(j)^{*}(d)[2 d]: M_{g m}(\bar{X})^{*}(d)[2 d] \longrightarrow M_{g m}(X)^{*}(d)[2 d],
$$

and of the duality isomorphism

$$
M_{g m}(X)^{*}(d)[2 d] \stackrel{\sim}{\longrightarrow} M_{g m}^{c}(X)
$$

V, Thm. 4.3.7 3]. Now recall that $R$ is compatible with the tensor structures $[\mathrm{Hu}$, Cor. 2.3.5, Cor. 2.3.4], and sends the Tate motive $\mathbb{Z}(1)$ to $\mathbb{Q}(-1)$ resp. $\mathbb{Q}_{\ell}(-1)$. It follows that $R$ is compatible with duality. Furthermore, $R$ sends $M_{g m}(f)$ to $f^{*}: R \Gamma(Z) \rightarrow R \Gamma(Y)$, for any morphism $f: Y \rightarrow Z$ of smooth $k$-schemes [DG, pp. 6-7]. Thus, $R$ sends $\iota$ to the composition of the duality isomorphism

$$
R \Gamma_{c}(X) \stackrel{\sim}{\longrightarrow} R \Gamma(X)^{*}(-d)[-2 d],
$$

the dual of $j^{*}$, the inverse of the duality isomorphism,

$$
R \Gamma(\bar{X})^{*}(-d)[-2 d] \stackrel{\sim}{\longrightarrow} R \Gamma(\bar{X}),
$$

and $j^{*}$. But this composition equals the canonical morphism

$$
R \Gamma_{c}(X) \longrightarrow R \Gamma(X) \text {. }
$$

It remains to show that the above identification is compatible with the action of $c_{1,2}(X, X)$. Let $\mathfrak{Z}$ be a cycle on $X \times_{k} X$ belonging to $c_{1,2}(X, X)$, and denote by ${ }^{t} \mathfrak{Z}$ its transpose. Both $\mathfrak{Z}$ and ${ }^{t} \mathfrak{Z}$ are finite over both components of $X \times X$. This is true in particular for the first component. Therefore, both induce endomorphisms of $M_{g m}(X)$. Similarly, they induce endomorphisms of $M_{g m}^{c}(X)$. Now the definition of the duality isomorphism

$$
M_{g m}(X)^{*}(d)[2 d] \stackrel{\sim}{\longrightarrow} M_{g m}^{c}(X)
$$

[V, proof of Thm. 4.3.7 3] implies that under this isomorphism, the endomorphism $\mathfrak{Z}$ of $M_{g m}^{c}(X)$ corresponds to the endomorphism ${ }^{t} \mathfrak{Z}^{*}(d)[2 d]$ of $M_{g m}(X)^{*}(d)[2 d]$. We thus identify the commutative diagram

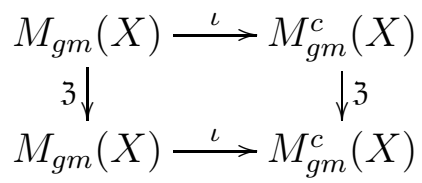

with

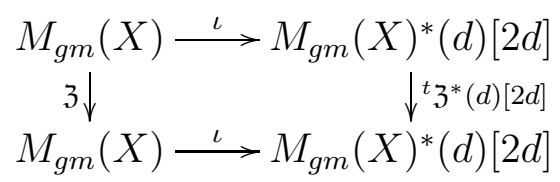


By [DG, pp. 6-7], $R$ sends $\mathfrak{Y}$ to $\mathfrak{Y}^{*}: R \Gamma(W) \rightarrow R \Gamma(V)$, for any finite correspondence $\mathfrak{Y}$ on the product $V \times_{k} W$ of two smooth $k$-schemes. It follows that $R$ sends the latter commutative diagram to the commutative diagram

$$
\begin{array}{cc}
R \Gamma(X) \longleftarrow R \Gamma(X)^{*}(-d)[-2 d] \\
\mathfrak{Z}^{*} \uparrow & \uparrow\left({ }^{t} \mathbf{Z}^{*}\right)^{*}(-d)[-2 d] \\
R \Gamma(X) \longleftarrow R \Gamma(X)^{*}(-d)[-2 d] & \longleftarrow R
\end{array}
$$

Now the endomorphism $\left({ }^{t} \mathfrak{Z}^{*}\right)^{*}(-d)[-2 d]$ of $R \Gamma(X)^{*}(-d)[-2 d]$ corresponds to the endomorphism $\mathfrak{Z}^{*}$ of $R \Gamma_{c}(X)$. But this means precisely that our identification of the image under $R$ of $M_{g m}(X) \rightarrow M_{g m}^{c}(X)$ with the canonical morphism $R \Gamma_{c}(X) \rightarrow R \Gamma(X)$ is $c_{1,2}(X, X)$-equivariant.

q.e.d.

Remark 2.6. In the Hodge theoretic setting, the isomorphism of mixed Hodge structures

$$
H^{n} R\left(\partial M_{g m}(X)\right) \cong\left(\partial H^{n}(X(\mathbb{C}), \mathbb{Q}) \otimes_{\mathbb{Q}} F\right)
$$

from Proposition 2.5 implies an isomorphism of $F$-modules. For quasiprojective $X$, this latter statement should be compared to [Ay, Lemme 3.12].

\section{Statement of the main results}

In order to state our main results (Theorems 3.5, 3.6), let us introduce the geometrical situation we are going to consider from now on. The base $k$ is the field $\mathbb{Q}$ of rational numbers, $X$ is the $r$-th power of the universal Abelian scheme over a Hilbert-Blumenthal variety of dimension $g$, and $e$ is associated to modular forms of weight $\left(r_{1}+2, \ldots, r_{g}+2\right)$, for $r_{1}+\ldots+r_{g}=r$ (see below for the precise definition). Theorems 3.5 and 3.6 imply in particular that in this context, Assumption 2.1 is satisfied as soon as $r \geq 1$ : indeed, $\partial M_{g m}(X)$ lies in $D M A T(\mathbb{Q})_{\mathbb{Q}}$, and the $e$-part of the $n$-th boundary cohomology group of $X$ is without weights $n-(r-1), \ldots, n+r$, for all $n \in \mathbb{Z}$. We then list the main consequences of this result (Corollaries [3.7, 3.8, 3.9, 3.13, 3.14), applying the theory developed in [W4, Sect. 4]. The proofs of Theorems 3.5 and 3.6 will be given in Section 4.

Fix a totally real number field $L$ of degree $g$. Let $I_{L}$ denote the set of real embeddings of $L$. Denote by $\operatorname{Res}_{L / \mathbb{Q}}$ the Weil restriction from schemes over $L$ to schemes over $\mathbb{Q}$. The functor $\operatorname{Res}_{L / \mathbb{Q}}$ is right adjoint to the base change $Z \mapsto Z_{L}:=Z \times_{\mathbb{Q}} L$. Hence we have in particular a functorial adjunction morphism $Z \rightarrow \operatorname{Res}_{L / \mathbb{Q}} Z_{L}$ for any scheme $Z$ over $\mathbb{Q}$. For any scheme $Y$ over 
$L$, and any subfield $F$ of $\mathbb{C}$ containing the images $\sigma(L)$ for all $\sigma \in I_{L}$, there is a canonical isomorphism

$$
\left(\operatorname{Res}_{L / \mathbb{Q}} Y\right) \times_{\mathbb{Q}} F \stackrel{\sim}{\longrightarrow} \prod_{\sigma \in I_{L}} Y \times_{L, \sigma} F
$$

induced by the isomorphism

$$
L \otimes_{\mathbb{Q}} R \stackrel{\sim}{\longrightarrow} \prod_{\sigma \in I_{L}} R, l \otimes r \longmapsto(\sigma(l) \cdot r)_{\sigma}
$$

for any $F$-algebra $R$. The composition of the adjunction (base changed by $F)$ and this isomorphism is simply the diagonal

$$
X \times_{\mathbb{Q}} F \longrightarrow \prod_{\sigma \in I_{L}} X \times_{\mathbb{Q}} F .
$$

By functoriality, the determinant induces a morphism of group schemes over $\mathbb{Q}$

$$
\text { det }: \operatorname{Res}_{L / \mathbb{Q}} \mathrm{GL}_{2, L} \longrightarrow \operatorname{Res}_{L / \mathbb{Q}} \mathbb{G}_{m, L}
$$

Definition 3.1 ([ $\mathbb{R}$, Sect. 1.27]). The group scheme $G$ over $\mathbb{Q}$ is defined as the fibre product

$$
G:=\mathbb{G}_{m} \times_{\operatorname{Res}_{L / \mathbb{Q}} \mathbb{G}_{m, L}, \operatorname{det}} \operatorname{Res}_{L / \mathbb{Q}} \mathrm{GL}_{2, L} .
$$

In particular, we have

$$
G(\mathbb{Q})=\left\{M \in \mathrm{GL}_{2}(L), \operatorname{det}(M) \in \mathbb{Q}^{*} \subset L^{*}\right\} .
$$

Under the above isomorphism

$$
\left(\operatorname{Res}_{L / \mathbb{Q}} \mathrm{GL}_{2, L}\right) \times_{\mathbb{Q}} \mathbb{R} \stackrel{\sim}{\longrightarrow} \prod_{\sigma \in I_{L}} \mathrm{GL}_{2, \mathbb{R}},
$$

we can identify

$$
G(\mathbb{R}) \cong\left\{\left(M_{\sigma}\right)_{\sigma \in I_{L}} \in \prod_{\sigma \in I_{L}} \mathrm{GL}_{2}(\mathbb{R}), \operatorname{det}\left(M_{\sigma}\right)=\operatorname{det}\left(M_{\eta}\right) \forall \sigma, \eta \in I_{L}\right\} .
$$

In particular, we see that $G(\mathbb{R})$ has two connected components, according to the sign of the determinant. Under these identifications, the inclusion of $G(\mathbb{Q})$ into $G(\mathbb{R})$ maps $M \in \mathrm{GL}_{2}(L)$ to the $g$-tuple

$$
(\sigma(M))_{\sigma \in I_{L}} \in \prod_{\sigma \in I_{L}} \mathrm{GL}_{2}(\mathbb{R}) .
$$

Definition 3.2. (a) The analytic space $\mathcal{H}$ is defined as

$$
\mathcal{H}:=\left\{\left(\tau_{\sigma}\right)_{\sigma \in I_{L}} \in \prod_{\sigma \in I_{L}}(\mathbb{C}-\mathbb{R}), \operatorname{sign}\left(\operatorname{im} \tau_{\sigma}\right)=\operatorname{sign}\left(\operatorname{im} \tau_{\eta}\right) \forall \sigma, \eta \in I_{L}\right\} .
$$


(b) The action of $G(\mathbb{R})$ on $\mathcal{H}$ is given by the usual componentwise action of $\mathrm{GL}_{2}(\mathbb{R})$ on $\mathbb{C}-\mathbb{R}$, and the above identification of $G(\mathbb{R})$ with a subgroup of $\prod_{\sigma \in I_{L}} \mathrm{GL}_{2}(\mathbb{R})$.

Given that the action of $\mathrm{GL}_{2}(\mathbb{R})$ on $\mathbb{C}-\mathbb{R}$ is transitive and trivial on $\mathbb{G}_{m}(\mathbb{R}) \subset \mathrm{GL}_{2}(\mathbb{R})$, it is easy to see that $G(\mathbb{R})$ acts transitively on $\mathcal{H}$. Observe that this action is by analytical automorphisms. In fact, $(G, \mathcal{H})$ are pure Shimura data $[\mathrm{P}$, Def. 2.1]. Their reflex field [P, Sect. 11.1] equals $\mathbb{Q}$. The center $Z(G)$ of $G$ equals

$$
\mathbb{G}_{m} \times \operatorname{Res}_{L / \mathbb{Q}} \mathbb{G}_{m, L}, x \mapsto x^{2} \operatorname{Res}_{L / \mathbb{Q}} \mathbb{G}_{m, L},
$$

hence its neutral connected component is isogeneous to $\mathbb{G}_{m}$. In particular, the Shimura data $(G, \mathcal{H})$ satisfy condition $(+)$ from [W3, Sect. 5].

Let us now fix additional data: (A) an open compact subgroup $K$ of $G\left(\mathbb{A}_{f}\right)$ which is neat $[\mathrm{P}$, Sect. 0.6], (B) a subfield $F$ of $\mathbb{C}$ containing the images of all embeddings $\sigma \in I_{L},(\mathrm{C})$ an integer $r \geq 0$, together with a partition

$$
\underline{r}: \quad r=\sum_{\sigma \in I_{L}} r_{\sigma}
$$

with $g$ integers $r_{\sigma} \geq 0$. Equivalently, we may see $\underline{r}=\sum_{\sigma} r_{\sigma} \cdot \sigma$ as an element of the free Abelian group $\mathbb{Z}\left[I_{L}\right]$ on $I_{L}$.

These data (A)-(C) are used as follows (cmp. [K, Sect. 2.2, 2.3] for the case $g=2)$. The Shimura variety $S:=S^{K}(G, \mathcal{H})$ is an object of $S m / \mathbb{Q}$. This is the Hilbert-Blumenthal variety of level $K$ associated to $L$. It is of dimension $g$, and admits an interpretation as modular space of Abelian varieties of dimension $g$ with additional structures, among which a real multiplication by a sub-algebra of $L$ which is of rank $g$ over $\mathbb{Z}$, hence of finite index in the ring of integers $\mathcal{O}_{L}$ (and which depends on $K$ ). In particular, there is a universal family $A$ of Abelian varieties over $S$. Thus, the absolute dimension of $A$ over $\mathbb{Q}$ is $2 g$, it is an object of $S m / \mathbb{Q}$, and thanks to the modular interpretation there is a canonical ring monomorphism

$$
L \hookrightarrow \operatorname{End}_{S}(A) \otimes_{\mathbb{Z}} \mathbb{Q}
$$

from $L$ to the endomorphisms of $A$ over $S$, tensored with $\mathbb{Q}$. Denote by $C H M(S)$ the category of Chow motives over $S$ [DeM, Sect. 1.3., 1.6]. The decomposition of the Chow motive of $A$ over $S$

$$
h(A / S)=\bigoplus_{i} h_{i}(A / S)
$$

[DeM, Thm. 3.1] being functorial, there is a map

$$
\operatorname{End}_{S}(A) \otimes_{\mathbb{Z}} \mathbb{Q} \longrightarrow \operatorname{End}_{C H M(S)}\left(h_{i}(A / S)\right) \otimes_{\mathbb{Z}} \mathbb{Q}
$$


for all $0 \leq i \leq 2 g$. For $i=1$, this map is an isomorphism of $\mathbb{Q}$-vector spaces [K, Prop. 2.2.1]. Hence, we get a ring monomorphism

$$
L \hookrightarrow \operatorname{End}_{C H M(S)}\left(h_{1}(A / S)\right) \otimes_{\mathbb{Z}} \mathbb{Q} .
$$

Its tensor product with $F$ gives

$$
L \otimes_{\mathbb{Q}} F \longleftrightarrow \operatorname{End}_{C H M(S)}\left(h_{1}(A / S)\right) \otimes_{\mathbb{Z}} F .
$$

The field $F$ containing the images of all $\sigma \in I_{L}$, we get canonically

$$
L \otimes_{\mathbb{Q}} F \stackrel{\sim}{\longrightarrow} \prod_{\sigma \in I_{L}} F, l \otimes f \longmapsto(\sigma(l) \cdot f)_{\sigma} .
$$

In particular, there are canonical idempotents $e_{\sigma}$ in $L \otimes_{\mathbb{Q}} F$, indexed by $I_{L}$ : by definition, $e_{\sigma}$ is the projection to the copy of $F$ corresponding to $\sigma$. Let us use the same symbol $e_{\sigma}$ for its image in

$$
\operatorname{End}_{C H M(S)}\left(h_{1}(A / S)\right) \otimes_{\mathbb{Z}} F \subset \mathrm{CH}^{g}\left(A \times_{S} A\right) \otimes_{\mathbb{Z}} F .
$$

From our construction, the relation

$$
(\Delta)=\sum_{\sigma \in I_{L}} e_{\sigma} \in \mathrm{CH}^{g}\left(A \times_{S} A\right) \otimes_{\mathbb{Z}} F
$$

is obvious. It induces a decomposition

$$
h_{1}(A / S)=\bigoplus_{\sigma \in I_{L}} h_{1}(A / S)^{e_{\sigma}}
$$

in $C H M(S)_{F}$, where $h_{1}(A / S)^{e_{\sigma}}$ denotes the image of the projector $e_{\sigma}$ on $h_{1}(A / S)$.

Let us now use the partition $\underline{r}=\sum_{\sigma} r_{\sigma} \cdot \sigma \in \mathbb{Z}\left[I_{L}\right]$.

Definition 3.3. Define $\stackrel{r}{\mathcal{V}} \in C H M(S)_{F}$ as

$$
\underline{r} \mathcal{V}:=\bigotimes_{\sigma \in I_{L}} \operatorname{Sym}^{r_{\sigma}} h_{1}(A / S)^{e_{\sigma}}
$$

The tensor product is in $C H M(S)_{F}$, and the symmetric powers are formed with the usual convention concerning the (twist of) the natural action of the symmetric group on a power of $A$ over $S$ (see e.g. [K, p. 72]). Thus, $\underline{r} \mathcal{V}$ is a direct factor of $h\left(A^{r} / S\right)$, where $A^{r}$ denotes the $r$-fold fibre product of $A$ over $S$. That is, it is associated to an idempotent

$$
e_{\underline{r}} \in \mathrm{CH}^{r g}\left(A^{r} \times{ }_{S} A^{r}\right) \otimes_{\mathbb{Z}} F .
$$

Let $c_{S}\left(A^{r}, A^{r}\right)$ denote the subgroup of $c\left(A^{r}, A^{r}\right)$ of correspondences whose support is contained in $A^{r} \times{ }_{S} A^{r} \subset A^{r} \times{ }_{k} A^{r}$. Define

$$
\mathrm{CH}^{r g}\left(A^{r} \times_{S} A^{r}\right)_{1,2} \subset \mathrm{CH}^{r g}\left(A^{r} \times{ }_{S} A^{r}\right)
$$


as the image of

$$
c_{S}\left(A^{r}, A^{r}\right) \cap c_{1,2}\left(A^{r}, A^{r}\right) \longrightarrow \mathrm{CH}^{r g}\left(A^{r} \times_{S} A^{r}\right) .
$$

Lemma 3.4. The idempotent $e_{\underline{r}}$ lies in

$$
\mathrm{CH}^{r g}\left(A^{r} \times{ }_{S} A^{r}\right)_{1,2} \otimes_{\mathbb{Z}} F \subset \mathrm{CH}^{r g}\left(A^{r} \times{ }_{S} A^{r}\right) \otimes_{\mathbb{Z}} F .
$$

Proof. $\quad$ By [W7, Prop. 3.4],

$$
\pi_{A, 1, n}:=\prod_{j \neq 1} \frac{\Gamma_{[n]_{A}}-n^{j}}{n-n^{j}}
$$

is a pre-image of $p_{A, 1}$ in $c_{S}(A, A) \otimes_{\mathbb{Z}} \mathbb{Q}$, for any $n \neq-1,0,1$. It visibly lies in the intersection $\left(c_{S}\left(A^{r}, A^{r}\right) \cap c_{1,2}\left(A^{r}, A^{r}\right)\right) \otimes_{\mathbb{Z}} \mathbb{Q}$.

Similarly, $e_{\sigma}$ is seen to be the image of the composition of $\pi_{A, 1, n}$ and

$$
\prod_{\tau \neq \sigma} \frac{\Gamma_{\alpha(l)}-\tau(l)}{\sigma(l)-\tau(l)},
$$

for any $l$ generating $L$ over $\mathbb{Q}$, and such that $\alpha(l)$ is a genuine endomorphism of $A$. The graph $\Gamma_{\alpha(l)}$ is a cycle in $A \times_{S} A$ which maps isomorphically to the first component of $A \times_{S} A$. Over the second component, it is necessarily finite: indeed, the element $l$ is invertible in $L$, hence $\alpha(l)$ in invertible in $\operatorname{End}_{S}(A) \otimes_{\mathbb{Z}} \mathbb{Q}$. Altogether, this proves that $e_{\sigma} \in \mathrm{CH}^{g}\left(A \times_{S} A\right) \otimes_{\mathbb{Z}} F$ comes from

$$
\left(c_{S}(A, A) \cap c_{1,2}(A, A)\right) \otimes_{\mathbb{Z}} F .
$$

The same is then true for the external product of the $e_{\sigma}$ corresponding to the direct factor

$$
\bigotimes_{\sigma \in I_{L}}\left(h_{1}(A / S)^{e_{\sigma}}\right)^{\otimes r_{\sigma}}
$$

of $h\left(A^{r} / S\right)$.

In order to get a pre-image of the idempotent $e_{\underline{r}}$, it suffices to take a suitable average over the action of a suitable finite group (a product of symmetric groups).

q.e.d.

According to [W7, Cor. 2.12], the idempotent $e_{\underline{r}}$ thus maps to an idempotent in $\bar{c}_{1,2}\left(A^{r}, A^{r}\right) \otimes_{\mathbb{Z}} F$. It will be denoted by the same symbol $e_{\underline{r}}$. By functoriality [W7, Thm. 2.2 (a)], the relative Chow motive

$$
\underline{r} \mathcal{V}=h\left(A^{r} / S\right)^{e_{r}}
$$

gives rise to an exact triangle

$$
\partial M_{g m}\left(A^{r}\right)^{e_{\underline{r}}} \longrightarrow M_{g m}\left(A^{r}\right)^{e_{\underline{r}}} \longrightarrow M_{g m}^{c}\left(A^{r}\right)^{e_{\underline{r}}} \longrightarrow \partial M_{g m}\left(A^{r}\right)^{e_{\underline{r}}}[1]
$$

in $D M_{g m}^{e f f}(k)_{F}$. Here are our main results. 
Theorem 3.5. The boundary motive $\partial M_{g m}\left(A^{r}\right)$ lies in the triangulated sub-category $D M D T(\mathbb{Q})_{\mathbb{Q}}$ of $D M_{g m}(\mathbb{Q})_{\mathbb{Q}}$ of Dirichlet-Tate motives over $\mathbb{Q}$ [W5, Def. $3.5(b)]$. Its direct factor $\partial M_{g m}\left(A^{r}\right)^{e_{r}}$ is without weights

$$
-r,-(r-1), \ldots, r-1 \text {. }
$$

In particular, Assumption 2.1 holds for $\partial M_{g m}\left(A^{r}\right)^{e_{\underline{r}}}$ whenever $r \geq 1$.

Theorem 3.6. Assume that there are $\tau, \sigma \in I_{L}$ such that $r_{\tau} \neq r_{\sigma}$ (hence $g \geq 2$ and $r \geq 1)$. Then $\partial M_{g m}\left(A^{r}\right)^{e_{r}}=0$, and $M_{g m}\left(A^{r}\right)^{e_{\underline{r}}} \cong M_{g m}^{c}\left(A^{r}\right)^{e^{e_{r}}}$ are effective Chow motives.

Theorems 3.5 and 3.6 will be proved in Section 4. Let us give their main corollaries, assuming that $r \geq 1$. First, we fix a weight filtration

$$
C_{\leq-(r+1)} \longrightarrow \partial M_{g m}\left(A^{r}\right)^{e_{r}} \longrightarrow C_{\geq r} \longrightarrow C_{\leq-(r+1)}[1]
$$

avoiding weights $-r, \ldots, r-1\left[\mathrm{~W} 4\right.$, Def. 1.6]. The category $\operatorname{DMDT}(\mathbb{Q})_{\mathbb{Q}}$ being pseudo-Abelian [W5, Cor. 2.6], the motives $C_{\leq-(r+1)}$ and $C_{\geq r}$ are DirichletTate motives over $\mathbb{Q}$ of weights $\leq-(r+1)$ and $\geq r$, respectively. Furthermore, $C_{\leq-(r+1)}=0=C_{\geq r}$ under the hypothesis of Theorem 3.6.

Corollary 3.7 ([W4, Thm. 4.3]). Assume $r \geq 1$.

(a) The motive $M_{g m}\left(A^{r}\right)^{e_{r}}$ is without weights $-r, \ldots,-1$, and the motive $M_{g m}^{c}\left(A^{r}\right)^{e_{\underline{r}}}$ is without weights $1, \ldots, r$. The Chow motives $\operatorname{Gr}_{0} M_{g m}\left(A^{r}\right)^{e_{\underline{r}}}$ and $\operatorname{Gr}_{0} M_{g m}^{c}\left(A^{r}\right)^{e_{r}}$ W4, Prop. 2.2] are defined, and they carry a natural action of

$$
\operatorname{GCen}_{\bar{c}_{1,2}\left(A^{r}, A^{r}\right)}\left(e_{\underline{r}}\right):=\left\{z \in \bar{c}_{1,2}\left(A^{r}, A^{r}\right) \otimes_{\mathbb{Z}} F, z e_{\underline{r}}=e_{\underline{r}} z e_{\underline{r}}\right\} .
$$

(b) There are canonical exact triangles

$$
C_{\leq-(r+1)} \longrightarrow M_{g m}\left(A^{r}\right)^{e_{\underline{r}}} \stackrel{\pi_{0}}{\longrightarrow} \operatorname{Gr}_{0} M_{g m}\left(A^{r}\right)^{e_{r}} \longrightarrow C_{\leq-(r+1)}[1]
$$

and

$$
C_{\geq r} \longrightarrow \operatorname{Gr}_{0} M_{g m}^{c}\left(A^{r}\right)^{e_{\underline{r}}} \stackrel{i_{0}}{\longrightarrow} M_{g m}^{c}\left(A^{r}\right)^{e_{\underline{r}}} \longrightarrow C_{\geq r}[1],
$$

which are stable under the natural action of $\operatorname{GCen}_{\bar{c}_{1,2}\left(A^{r}, A^{r}\right)}\left(e_{\underline{r}}\right)$.

(c) There is a canonical isomorphism $\mathrm{Gr}_{0} M_{g m}\left(A^{r}\right)^{e_{\underline{r}}} \stackrel{\sim}{\longrightarrow} \mathrm{Gr}_{0} M_{g m}^{c}\left(A^{r}\right)^{e_{\underline{r}}}$ in $C H M^{e f f}(k)_{F}$. As a morphism, it is uniquely determined by the property of making the diagram

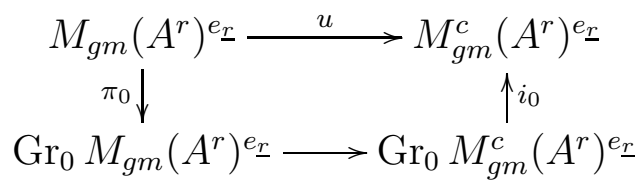

commute; in particular, it is $\mathrm{GCen}_{\bar{c}_{1,2}\left(A^{r}, A^{r}\right)}\left(e_{\underline{\underline{r}}}\right)$-equivariant.

(d) Let $N \in C H M(k)_{F}$ be a Chow motive. Then $\pi_{0}$ and $i_{0}$ induce isomorphisms

$$
\operatorname{Hom}_{C H M(k)_{F}}\left(\operatorname{Gr}_{0} M_{g m}\left(A^{r}\right)^{e_{\underline{r}}}, N\right) \stackrel{\sim}{\longrightarrow} \operatorname{Hom}_{D M_{g m}(k)_{F}}\left(M_{g m}\left(A^{r}\right)^{e_{\underline{r}}}, N\right)
$$


and

$$
\operatorname{Hom}_{C H M(k)_{F}}\left(N, \operatorname{Gr}_{0} M_{g m}^{c}\left(A^{r}\right)^{e_{\underline{r}}}\right) \stackrel{\sim}{\longrightarrow} \operatorname{Hom}_{D M_{g m}(k)_{F}}\left(N, M_{g m}^{c}\left(A^{r}\right)^{e_{\underline{r}}}\right) .
$$

(e) Let $M_{g m}\left(A^{r}\right)^{e_{\underline{r}}} \rightarrow N \rightarrow M_{g m}^{c}\left(A^{r}\right)^{e_{\underline{r}}}$ be a factorization of $u$ through a Chow motive $N \in C H M(k)_{F}$. Then $\operatorname{Gr}_{0} M_{g m}\left(A^{r}\right)^{e_{\underline{r}}}=\operatorname{Gr}_{0} M_{g m}^{c}\left(A^{r}\right)^{e_{\underline{r}}}$ is canonically a direct factor of $N$, with a canonical direct complement.

Henceforth, we identify $\operatorname{Gr}_{0} M_{g m}\left(A^{r}\right)^{e_{\underline{r}}}$ and $\mathrm{Gr}_{0} M_{g m}^{c}\left(A^{r}\right)^{e_{\underline{r}}}$ via the canonical isomorphism of Corollary 3.7 (c). Note that under the hypothesis of Theorem 3.6. we have

$$
M_{g m}\left(A^{r}\right)^{e_{\underline{r}}}=\operatorname{Gr}_{0} M_{g m}\left(A^{r}\right)^{e_{\underline{r}}} \text { and } \operatorname{Gr}_{0} M_{g m}^{c}\left(A^{r}\right)^{e_{\underline{r}}}=M_{g m}^{c}\left(A^{r}\right)^{e_{\underline{r}}},
$$

and the isomorphism of Corollary 3.7 (c) coincides with that of Theorem 3.6. The equivariance statements from Corollary 3.7 (a)-(c) apply in particular to cycles coming from the Hecke algebra associated to the Shimura variety $S$. More precisely, we have the following statement.

Corollary 3.8. Assume $r \geq 1$. Then $\mathrm{Gr}_{0} M_{g m}\left(A^{r}\right)^{e_{\underline{r}}}$ carries a natural action of the Hecke algebra $R\left(K, G\left(\mathbb{A}_{f}\right)\right)$ associated to the neat open compact subgroup $K$ of $G\left(\mathbb{A}_{f}\right)$. More precisely, any $x \in G\left(\mathbb{A}_{f}\right)$ defines a cycle denoted $K x K$ in $c_{1,2}\left(A^{r}, A^{r}\right)$, whose class in $\bar{c}_{1,2}\left(A^{r}, A^{r}\right)$ belongs to the centralizer

$\operatorname{Cen}_{\bar{c}_{1,2}\left(A^{r}, A^{r}\right)}\left(e_{\underline{r}}\right):=\left\{z \in \bar{c}_{1,2}\left(A^{r}, A^{r}\right) \otimes_{\mathbb{Z}} F, z e_{\underline{\underline{r}}}=e_{\underline{r}} z\right\} \subset G C e n_{\bar{c}_{1,2}\left(A^{r}, A^{r}\right)}\left(e_{\underline{r}}\right)$ of $e_{\underline{r}}$.

Proof. Fix $x \in G\left(\mathbb{A}_{f}\right)$. Recall that our base scheme $S$ equals the Hilbert-Blumenthal variety $S^{K}(G, \mathcal{H})$. It is the target of two finite étale morphisms $g_{1}, g_{2}: U \rightarrow S$, where $U$ denotes the Hilbert-Blumenthal variety $S^{K \cap x^{-1} K x}(G, \mathcal{H})$. Using the notation of [P, Sect. 3.4], the morphism $g_{1}$ equals $[\cdot 1]$, and the morphism $g_{2}$ equals $\left[\cdot x^{-1}\right]$. Note that by the very definition of the $\mathbb{Q}$-rational structure of $S$ and $U$ (e.g. [P, Def. 11.5]), both $g_{1}$ and $g_{2}$ are indeed defined over $\mathbb{Q}$.

Recall that $A$ is the universal Abelian scheme over $S$; denote by $A_{1}, A_{2}$ its base changes to $U$ via $g_{1}$ and $g_{2}$, respectively. To the data $K$ and $x$, the following are canonically associated: a third Abelian scheme $B$ over $U$ admitting real multiplication, and isogenies $f_{1}: B \rightarrow A_{1}$ and $f_{2}: B \rightarrow A_{2}$ compatible with the real multiplications. By definition, the cycle $K x K$ is then equal to the direct image under $g_{1} \times_{k} g_{2}$ of the composition

$$
\Gamma_{f_{2}^{r}} \circ{ }^{t} \Gamma_{f_{1}^{r}} \in c_{U}\left(A_{1}^{r}, A_{2}^{r}\right) \cap c_{1,2}\left(A_{1}^{r}, A_{2}^{r}\right)
$$

(= "pull-back via $f_{1}^{r}$ followed by push-out via $f_{2}^{r "}$ ).

In order to show that the class of $K x K$ in $\bar{c}_{1,2}\left(A^{r}, A^{r}\right)$ commutes with $e_{\underline{r}}$, note first that $\varphi:=\Gamma_{f_{2}^{r}} \circ{ }^{t} \Gamma_{f_{1}^{r}}$ defines a morphism of relative Chow motives over $U$,

$$
\varphi: h\left(A_{1}^{r} / U\right)=g_{1}^{*}\left(h\left(A^{r} / S\right)\right) \longrightarrow g_{2}^{*}\left(h\left(A^{r} / S\right)\right)=h\left(A_{2}^{r} / U\right) .
$$


Then, since both $f_{1}$ and $f_{2}$ are isogenies, this morphism is compatible with the external products of the idempotents $p_{A_{i}, 1}$ [DeM, Thm. 3.1, Prop. 3.3]. Since $f_{1}$ and $f_{2}$ also respect the real multiplication, the morphism $\varphi$ is also compatible with the cycle classes $g_{i}^{*}\left(e_{\underline{r}}\right)=e_{\underline{r}, i} \in \mathrm{CH}^{r g}\left(A_{i}^{r} \times_{U} A_{i}^{r}\right) \otimes_{\mathbb{Z}} F$, i.e., we have the relation

$$
\varphi \circ g_{1}^{*}\left(e_{\underline{r}}\right)=g_{2}^{*}\left(e_{\underline{r}}\right) \circ \varphi
$$

of morphisms of relative Chow motives over $U$. We are thus in the situation of [W7, Ex. 2.16 (d)]. Now observe that using the notation from [loc. cit.], the effect of the cycle $K x K$ on the exact triangle

$$
\partial M_{g m}\left(A^{r}\right) \longrightarrow M_{g m}\left(A^{r}\right) \stackrel{u}{\longrightarrow} M_{g m}^{c}\left(A^{r}\right) \longrightarrow \partial M_{g m}\left(A^{r}\right)[1]
$$

coincides with $\varphi\left(g_{1}, g_{2}\right)$ [W7, Ex. $\left.2.16(\mathrm{e})\right]$. Thanks to the relation $\varphi \circ g_{1}^{*}\left(e_{\underline{r}}\right)=$ $g_{2}^{*}\left(e_{\underline{r}}\right) \circ \varphi$, we thus get that the class of $K x K$ in $\bar{c}_{1,2}\left(A^{r}, A^{r}\right)$ belongs indeed to $\overline{C e n_{\bar{c}_{1,2}}\left(A^{r}, A^{r}\right)}\left(e_{\underline{r}}\right)$.

q.e.d.

Corollary 3.9 ([W4, Cor. 4.6]). Assume $r \geq 1$, and let $\widetilde{A^{r}}$ be any smooth compactification of $A^{r}$. Then $\operatorname{Gr}_{0} M_{g m}\left(A^{r}\right)^{e_{r}}$ is canonically a direct factor of the Chow motive $M_{g m}\left(\widetilde{A^{r}}\right)$, with a canonical direct complement.

Furthermore, [W4, Thm. 4.7, Thm. 4.8] on the Hodge theoretic and $\ell-$ adic realizations [ $\mathrm{Hu}$, Cor. 2.3.5, Cor. 2.3.4 and Corrigendum] apply, and tell us in particular that $\operatorname{Gr}_{0} M_{g m}\left(A^{r}\right)^{e_{r}}$ is mapped to the part of interior cohomology of $A^{r}$ fixed by $e_{\underline{r}}$. In particular, the $L$-function of the Chow motive $\operatorname{Gr}_{0} M_{g m}\left(A^{r}\right)^{e_{\underline{r}}}$ is computed via (the $e_{\underline{r}}$-part of) interior cohomology of $A^{r}$.

Definition 3.10 ([W4, Def. 4.9]). Let $r \geq 1$. We call $\mathrm{Gr}_{0} M_{g m}\left(A^{r}\right)^{e_{\underline{r}}}$ the $e_{\underline{r}}$-part of the interior motive of $A^{r}$.

Remark 3.11. By [W4, Thm. 4.14], control of the reduction of some compactification of $A^{r}$ implies control of certain properties of the $\ell$-adic realization of $\operatorname{Gr}_{0} M_{g m}\left(A^{r}\right)^{e_{r}}$. To the best of the author's knowledge, the sharpest result known about reduction of compactifications of $A^{r}$ is [DiT, Thm. 6.4]. It concerns the case when $K \subset G\left(\mathbb{A}_{f}\right)$ is of type $\Gamma_{1}$, and states that there exist then smooth compactifications of $A^{r}$ having good reduction at each prime number $p$ dividing neither the level $N$ of $K$ nor the absolute discriminant $d$ of L. [W4, Thm. 4.14] then yields the following conclusions: (a) for all primes $p$ not dividing $N d$, the $p$-adic realization of $\operatorname{Gr}_{0} M_{g m}\left(A^{r}\right)^{e_{\underline{r}}}$ is crystalline, (b) if furthermore $p \neq \ell$, then the $\ell$-adic realization of $\operatorname{Gr}_{0} M_{g m}\left(A^{r}\right)^{e_{\underline{r}}}$ is unramified. Note that given the identification of the $\ell$-adic realization of $\operatorname{Gr}_{0} M_{g m}\left(A^{r}\right)^{e_{r}}$ with intersection cohomology, conclusions (a) ane (b) are already contained in [DiT, Sect. 7].

Remark 3.12. (a) If all $r_{\sigma}$ are strictly positive (hence $r \geq g$ ) then Saper's vanishing theorem on (ordinary) cohomology [Sp, Thm. 5] implies that the 
realizations of $\operatorname{Gr}_{0} M_{g m}\left(A^{r}\right)^{e_{\underline{r}}}$ are concentrated in the single cohomological degree $r+g$. In particular, we expect the following relation to the ChowKünneth decompositions constructed in GHM2, Thm. 2.4]. The base change from $\mathbb{Q}$ to $\mathbb{C}$ of $\mathrm{Gr}_{0} M_{g m}\left(A^{r}\right)^{e_{r}}$ should map monomorphically to the $(r+g)$ th Chow-Künneth component of the motive (over $\mathbb{C}$ ) of any toroidal compactification of $A^{r}$.

(b) In general, consider the (relative) Chow-Künneth projectors $\Pi_{S}^{i}, i=$ $0, \ldots, 2 \mathrm{rg}$ of GHM1, Thm. I] modelling intersection cohomology of $S(\mathbb{C})$ with coefficients in the $i$-th higher direct image of the constant sheaf $\mathbb{Q}_{A^{r}}$. For $i=r \geq 1$, we expect the image of $\Pi_{S}^{r}$ to contain a copy of the base change to $\mathbb{C}$ of the interior motive $\mathrm{Gr}_{0} M_{g m}(X)^{e}$.

Let us discuss special cases. First, for $g=1$, we have $L=\mathbb{Q}, S$ is a smooth modular curve, and $A$ the universal family of elliptic curves over $S$ (see our Introduction). As coefficient field (B), we may choose $F=\mathbb{Q}$. The partition (C) amounts to fixing an integer $r \geq 0$, and $\stackrel{r}{V}=\operatorname{Sym}^{r} h_{1}(A / S)$. In this setting, the formal implications of Theorem 3.5 are discussed in [W4, Rem. 4.17]; note that our idempotent $e_{\underline{r}}$ coincides with the idempotent denoted $e$ in [loc. cit.]. Indeed, the additional action of torsion entering the definition of $e$ is known (and easily shown) to be trivial on the relative motive $h(A / S)$. In particular [W4, Rem. $4.17(\mathrm{~b})$ ], we get an alternative construction of the Grothendieck motive $M(f)$ associated to a normalized newform $f$ of weight $r+2[\mathrm{Sch}]$ as a direct factor of the Grothendieck motive underlying $\mathrm{Gr}_{0} M_{g m}\left(A^{r}\right)^{e_{\underline{r}}}$.

Now let $g=2$. Here, we have $[L: \mathbb{Q}]=2, S$ is a smooth HilbertBlumenthal surface, and $A$ the universal family of Abelian surfaces over $S$. As coefficient field (B), we may choose $F$ equal to the image of $L$ under any of its two real embeddings $\tau, \sigma \in I_{L}$. The partition (C) amounts to fixing two integers $r_{\tau}, r_{\sigma} \geq 0$, whose sum is denoted $r$. Then

$$
{ }^{\underline{V}} \mathcal{V}=\operatorname{Sym}^{r_{\tau}} h_{1}(A / S)^{e_{\tau}} \otimes \operatorname{Sym}^{r_{\sigma}} h_{1}(A / S)^{e_{\sigma}} .
$$

For any object $M$ of $D M_{g m}^{e f f}(\mathbb{Q})_{F}$, define motivic cohomology

$$
H_{\mathcal{M}}^{p}(M, F(q)):=\operatorname{Hom}_{D M_{g m}^{e f f}(\mathbb{Q})_{F}}(M, \mathbb{Z}(q)[p]) .
$$

When $M=M_{g m}(Y)$ for a scheme $Y \in S m / \mathbb{Q}$, this gives motivic cohomology $H_{\mathcal{M}}^{p}(Y, \mathbb{Z}(q))$ of $Y$, tensored with $F$. Now observe that the relative Chow motive $\underline{\underline{r}} \mathcal{V}$ coincides with the object denoted $\mathcal{V}_{K}^{r_{\tau}, r_{\sigma}}$ in [K, Def. 2.3.1]. From now on, assume that $r_{\tau} \geq r_{\sigma} \geq 1$ (hence $r \geq 2$ ). The main result of [loc. cit.] gives the construction of a sub-space

$$
\mathcal{K}\left(r_{\tau}, r_{\sigma}, n\right) \subset H_{\mathcal{M}}^{r+3}(\underline{r} \mathcal{V}, F(n))=\operatorname{Hom}_{D M_{g m}^{e f f}(\mathbb{Q})_{F}}\left(M_{g m}\left(A^{r}\right)^{e_{r}}, \mathbb{Z}(n)[r+3]\right)
$$

for all integers $n$ between $r_{\tau}+2$ and $r+2=r_{\tau}+r_{\sigma}+2$ [K, Thm. 5.2.4], and establishes a weak version of Beilinson's conjecture for Asai $L$-functions $\mathrm{K}$, Thm. 5.2.4 (b)]. As already mentioned in [K, p. 62, Rem. 5.2.5 (a)], one of 
the shortcomings of this result is that $\mathcal{K}\left(r_{\tau}, r_{\sigma}, n\right)$ is not shown to come from motivic cohomology of a smooth compactification of $A^{r}$. It is reasonable to expect this to be true; one of the indications being $[\mathrm{K}$, Thm. 5.2.4 (a)] that the Hodge theoretic realization of $\mathcal{K}\left(r_{\tau}, r_{\sigma}, n\right)$ lands in

$$
H_{! \mathfrak{H}}^{r+3}\left(\underline{r}^{-} \mathcal{V}_{\mathbb{R}}, \mathbb{R}(n)\right) \subset H_{\mathfrak{H}}^{r+3}\left({ }^{\underline{r}} \mathcal{V}_{/ \mathbb{R}}, \mathbb{R}(n)\right)
$$

which by definition [K, Def. (2.4.1)] is the sub-space of absolute Hodge cohomology of $\stackrel{r}{V} / \mathbb{R}$ given by the image of absolute Hodge cohomology of any smooth compactification of $A^{r}$. Our main results allow to give a significantly more precise statement.

Corollary 3.13. Assume that $r_{\tau} \geq r_{\sigma}+1(\geq 2)$ or that $r_{\tau}+2 \leq n \leq r+1$. Then the map on the level of motivic cohomology induced by the morphism $\pi_{0}: M_{g m}\left(A^{r}\right)^{e_{r}} \rightarrow \operatorname{Gr}_{0} M_{g m}\left(A^{r}\right)^{e_{\underline{r}}}$,

$$
\pi_{0}^{*}: \operatorname{Hom}_{D M_{g m}(\mathbb{Q})_{F}}\left(\operatorname{Gr}_{0} M_{g m}\left(A^{r}\right)^{e_{r}}, \mathbb{Z}(n)[r+3]\right) \longrightarrow H_{\mathcal{M}}^{r+3}(\underline{r} \mathcal{V}, F(n))
$$

is an isomorphism.

In particular, under the hypotheses of the corollary, $\mathcal{K}\left(r_{\tau}, r_{\sigma}, n\right)$ can be considered as a sub-space of $\operatorname{Hom}_{D M_{g m}(\mathbb{Q})_{F}}\left(\operatorname{Gr}_{0} M_{g m}\left(A^{r}\right)^{e_{r}}, \mathbb{Z}(n)[r+3]\right)$, and hence (Corollary 3.9) of motivic cohomology of any smooth compactification of $A^{r}$.

Corollary 3.14. Assume that $r_{\tau}=r_{\sigma}(\geq 1)$, hence $r=2 r_{\tau}$. Then the image of the map on the level of motivic cohomology induced by $\pi_{0}$,

$$
\pi_{0}^{*}\left(\operatorname{Hom}_{D M_{g m}(\mathbb{Q})_{F}}\left(\operatorname{Gr}_{0} M_{g m}\left(A^{r}\right)^{e_{\underline{r}}}, \mathbb{Z}(r+2)[r+3]\right)\right) \subset H_{\mathcal{M}}^{r+3}(\underline{r} \mathcal{V}, F(r+2))
$$

contains the sub-space $\mathcal{K}\left(r_{\tau}, r_{\sigma}, r+2\right)$.

In particular, under the hypotheses of the corollary, $\mathcal{K}\left(r_{\tau}, r_{\sigma}, r+2\right)$ comes from a sub-space of $\operatorname{Hom}_{D M_{g m}(\mathbb{Q})_{F}}\left(\operatorname{Gr}_{0} M_{g m}\left(A^{r}\right)^{e_{r}}, \mathbb{Z}(r+2)[r+3]\right)$.

Remark 3.15. This settles the problem raised in [K, Rem. 5.2.5 (a)]. At least two other points remain, in order to get a proof of Beilinson's full conjecture: first [K, Rem. 5.2.5 (c)], the elements in $\mathcal{K}\left(r_{\tau}, r_{\sigma}, n\right)$ should be integral (with respect to suitable models over Spec $\mathbb{Z}$ ), second, the space of integral elements in motivic cohomology should be equal to $\mathcal{K}\left(r_{\tau}, r_{\sigma}, n\right)$. We have nothing to say about these two points.

Proof of Corollaries 3.13 and 3.14. Recall the exact triangle

$$
C_{\leq-(r+1)} \longrightarrow M_{g m}\left(A^{r}\right)^{e_{r}} \stackrel{\pi_{0}}{\longrightarrow} \operatorname{Gr}_{0} M_{g m}\left(A^{r}\right)^{e_{r}} \longrightarrow C_{\leq-(r+1)}[1]
$$

from Corollary 3.7 (b). Here, $C_{\leq-(r+1)}$ is a Dirichlet-Tate motive over $\mathbb{Q}$ of weights $\leq-(r+1)$. Theorem 3.6 tells us that

$$
C_{\leq-(r+1)}=0
$$


when $r_{\tau} \geq r_{\sigma}+1$. In this case, the morphism $\pi_{0}$ is therefore itself an isomorphism, and thus induces an isomorphism on the level of motivic cohomology.

In general, the kernel of

$$
\pi_{0}^{*}: \operatorname{Hom}_{D M_{g m}(\mathbb{Q})_{F}}\left(\operatorname{Gr}_{0} M_{g m}\left(A^{r}\right)^{e_{r}}, \mathbb{Z}(n)[r+3]\right) \longrightarrow H_{\mathcal{M}}^{r+3}(\underline{r} \mathcal{V}, F(n))
$$

is a quotient of

$\operatorname{Hom}_{D M_{g m}(\mathbb{Q})_{F}}\left(C_{\leq-(r+1)}[1], \mathbb{Z}(n)[r+3]\right)=\operatorname{Hom}_{D M_{g m}(\mathbb{Q})_{F}}\left(C_{\leq-(r+1)}, \mathbb{Z}(n)[r+2]\right)$

and the co-kernel a sub-space of

$$
\operatorname{Hom}_{D M_{g m}(\mathbb{Q})_{F}}\left(C_{\leq-(r+1)}, \mathbb{Z}(n)[r+3]\right) .
$$

Now $\mathbb{Z}(n)$ is pure of weight $-2 n$. When $n \leq r+1$, then the weights of $\mathbb{Z}(n)[r+2]$ and of $\mathbb{Z}(n)[r+3]$ are at least equal to $-2(r+1)+r+2=-r$. Since $C_{\leq-(r+1)}$ is of weights at most $-(r+1)$, orthogonality [Bo, Def. 1.1.1 (iii)] of the motivic weight structure $[\mathrm{BO}$, Sect. 6.5 and 6.6] implies that both $\operatorname{Hom}_{D M_{g m}(\mathbb{Q})_{F}}\left(C_{\leq-(r+1)}, \mathbb{Z}(n)[r+2]\right)$ and $\operatorname{Hom}_{D M_{g m}(\mathbb{Q})_{F}}\left(C_{\leq-(r+1)}, \mathbb{Z}(n)[r+3]\right)$ are zero. In this case, the map $\pi_{0}^{*}$ is therefore again an isomorphism.

In the sequel, let us therefore assume that $r_{\tau}=r_{\sigma}$, and that $n=r+2$. As above, the co-kernel injects into

$$
\operatorname{Hom}_{D M_{g m}(\mathbb{Q})_{F}}\left(C_{\leq-(r+1)}, \mathbb{Z}(n)[r+3]\right) .
$$

Let us first show that on this latter space, the map induced by the Hodge theoretic realization is injective. Observe that the motive $\mathbb{Z}(n)[r+3]$ is pure of weight $-(r+1)$. This is the highest weight possibly occurring in $C_{\leq-(r+1)}$. Shifting by $r+1$ therefore reduces us to show the following: for any two Dirichlet-Tate motives $M$ and $N$, with $M \in D M D T(\mathbb{Q})_{F, w \leq 0}$ and $N \in D M D T(\mathbb{Q})_{F, w=0}$, the map induced on

$$
\operatorname{Hom}_{D M_{g m}(\mathbb{Q})_{F}}(M, N)
$$

by the Hodge theoretic realization is injective. Choose an exact triangle

$$
M_{\leq-2} \longrightarrow M \longrightarrow M_{-1,0} \longrightarrow M_{\leq-2}[1] \text {, }
$$

with $M_{\leq-2} \in \operatorname{DMDT}(\mathbb{Q})_{F, w \leq-2}$ and

$$
M_{-1,0} \in \operatorname{DMDT}(\mathbb{Q})_{F, w \geq-1} \cap \operatorname{DMDT}(\mathbb{Q})_{F, w \leq 0} .
$$

By [W5, Cor. 2.9], the object $M_{-1,0}$ is a direct sum of two Dirichlet-Tate motives $M_{-1} \oplus \mathrm{Gr}_{0} M$, the first being pure of weight -1 and the second pure of weight 0 . Orthogonality formally implies that the two morphisms $M \rightarrow M_{-1,0}$ and $M_{-1,0} \rightarrow \mathrm{Gr}_{0} M$ induce isomorphisms

$$
\operatorname{Hom}_{D M_{g m}(\mathbb{Q})_{F}}\left(M_{-1,0}, N\right) \stackrel{\sim}{\longrightarrow} \operatorname{Hom}_{D M_{g m}(\mathbb{Q})_{F}}(M, N)
$$

and

$$
\operatorname{Hom}_{D M_{g m}(\mathbb{Q})_{F}}\left(\operatorname{Gr}_{0} M, N\right) \stackrel{\sim}{\longrightarrow} \operatorname{Hom}_{D M_{g m}(\mathbb{Q})_{F}}\left(M_{-1,0}, N\right)
$$


The Hodge theoretic realization $R$ maps our data to an exact triangle

$$
R\left(M_{\leq-2}\right) \longleftarrow R(M) \longleftarrow R\left(M_{-1,0}\right) \longleftarrow R\left(M_{\leq-2}\right)[-1]
$$

in the bounded derived category $D$ of mixed graded-polarizable Hodge structures (recall that $R$ is contravariant), and a direct sum decomposition

$$
R\left(M_{-1}\right) \oplus R\left(\operatorname{Gr}_{0} M\right)=R\left(M_{-1,0}\right) .
$$

According to [W5, Rem. 3.13 (a)], the functor $R$ respects the weight structures. This means that $R\left(M_{\leq-2}\right)$ has weights at least 2 , that $R\left(M_{-1}\right)$ is pure of weight 1 and that $R\left(\mathrm{Gr}_{0} \bar{M}\right)$ and $R(N)$ are pure of weight 0 . As above, orthogonality (for the category $D$ ) yields formally that

$$
\operatorname{Hom}_{D}\left(K, R\left(M_{-1,0}\right)\right) \stackrel{\sim}{\longrightarrow} \operatorname{Hom}_{D}(K, R(M))
$$

and

$$
\operatorname{Hom}_{D}\left(K, R\left(\operatorname{Gr}_{0} M\right)\right) \stackrel{\sim}{\longrightarrow} \operatorname{Hom}_{D}\left(K, R\left(M_{-1,0}\right)\right)
$$

for any object $K$ of the heart $D_{w=0}$, hence in particular for $K=R(N)$. Altogether, we are thus reduced to showing injectivity of

$$
R: \operatorname{Hom}_{D M_{g m}(\mathbb{Q})_{F}}(M, N) \longrightarrow \operatorname{Hom}_{D}(R(N), R(M))
$$

under the additional assumption that $M$ belongs to the heart $\operatorname{DMT}(\mathbb{Q})_{F, w=0}$, too. In other words, we must show faithfulness of the restriction of $R$ to $\operatorname{DMDT}(\mathbb{Q})_{F, w=0}$. But this follows easily from the explicit description of $\operatorname{DMDT}(\mathbb{Q})_{F, w=0}$ given in $[\mathrm{W} 5$, Thm. 2.5 (c)], and the (obvious) faithfulness of $R$ on the category $M D(k)_{F}$ from [W5, Def. 3.5 (a)].

In order to finish the proof, it remains to show that the image of the space $\mathcal{K}\left(r_{\tau}, r_{\sigma}, n\right)$ in

$$
\operatorname{Hom}_{D M_{g m}(\mathbb{Q})_{F}}\left(C_{\leq-(r+1)}, \mathbb{Z}(n)[r+3]\right)
$$

is mapped to zero under $R$. Choose a smooth compactification $\widetilde{A^{r}}$ of $A^{r}$. By Corollary 3.7 (d), the morphism $j: M_{g m}\left(A^{r}\right)^{e_{r}} \rightarrow M_{g m}\left(A^{r}\right) \rightarrow M_{g m}\left(\widetilde{A^{r}}\right)$ factors through $\mathrm{Gr}_{0} M_{g m}\left(A^{r}\right)^{e_{\underline{r}}}$. It follows that the exact triangle

$$
C_{\leq-(r+1)} \longrightarrow M_{g m}\left(A^{r}\right)^{e_{\underline{r}}} \longrightarrow \mathrm{Gr}_{0} M_{g m}\left(A^{r}\right)^{e_{\underline{r}}} \longrightarrow C_{\leq-(r+1)}[1]
$$

maps to an exact triangle of the form

$$
C^{\prime} \stackrel{i}{\longrightarrow} M_{g m}\left(A^{r}\right)^{e_{\underline{r}}} \stackrel{j}{\longrightarrow} M_{g m}\left(\widetilde{A^{r}}\right) \longrightarrow C^{\prime}[1] .
$$

By [K, Thm. 5.2.4 (a)], the sub-space

$$
\mathcal{K}\left(r_{\tau}, r_{\sigma}, n\right) \subset \operatorname{Hom}_{D M_{g m}^{e f f}(\mathbb{Q})_{F}}\left(M_{g m}\left(A^{r}\right)^{e_{\underline{r}}}, \mathbb{Z}(n)[r+3]\right)
$$

vanishes under the composition of

$i^{*}: \operatorname{Hom}_{D M_{g m}^{e f f}(\mathbb{Q})_{F}}\left(M_{g m}\left(A^{r}\right)^{e_{r}}, \mathbb{Z}(n)[r+3]\right) \longrightarrow \operatorname{Hom}_{D M_{g m}^{e f f}(\mathbb{Q})_{F}}\left(C^{\prime}, \mathbb{Z}(n)[r+3]\right)$

and of $R$. A fortiori, its image in

$$
\operatorname{Hom}_{D M_{g m}(\mathbb{Q})_{F}}\left(C_{\leq-(r+1)}, \mathbb{Z}(n)[r+3]\right)
$$


vanishes under $R$.

q.e.d.

Remark 3.16. The picture for $r_{\tau}=r_{\sigma}$ and $n=r+2$ remains incomplete: it is clearly desirable to identify a canonical pre-image of $\mathcal{K}\left(r_{\tau}, r_{\sigma}, r+2\right)$ in

$$
\operatorname{Hom}_{D M_{g m}(\mathbb{Q})_{F}}\left(\operatorname{Gr}_{0} M_{g m}\left(A^{r}\right)^{e_{\underline{r}}}, \mathbb{Z}(r+2)[r+3]\right) \text {. }
$$

In order to achieve this, one needs to go into the construction of the elements from $[\mathrm{K}]$. The vital ingredient is Beilinson's Eisenstein symbol [Be1], which needs to be re-interpreted in the context of the category $D M_{g m}(\mathbb{Q})_{F}$. We plan to treat this elsewhere.

Remark 3.17. The case $r=0$ is not covered by our main results. It concerns the motive and the motive with compact support of the base scheme $S$. In this situation, the best replacement of the interior motive is the (hypothetical) intersection motive $M^{! *}(S)$ of $S$ with respect to its Baily-Borel compactification. Note that for $g \leq 2$, its existence is established: for modular curves $(g=1)$, it equals the motive of the smooth compactification $S^{*}$ of $S$. For $g=2$, we refer to [CMi, W6].

\section{Proof of the main results}

We keep the notation of the preceding section. In order to prove Theorems 3.5 and 3.6, our idea is to apply the criterion from Section 2. Let us start by fixing some notation.

Definition 4.1. Denote by $V$ the standard two-dimensional representation of $\mathrm{GL}_{2, L}$ over $L$.

Thus, $\operatorname{Res}_{L / \mathbb{Q}} V$ is a $2 g$-dimensional representation of $\operatorname{Res}_{L / \mathbb{Q}} \mathrm{GL}_{2, L}$, and therefore, of $G$.

Theorem 4.2. For any integer $r \geq 0$, the boundary motive $\partial M_{g m}\left(A^{r}\right)$ lies in the triangulated sub-category $\operatorname{DMDT}(\mathbb{Q})_{\mathbb{Q}}$ of $D M_{g m}(\mathbb{Q})_{\mathbb{Q}}$ of DirichletTate motives over $\mathbb{Q}$.

Proof. The variety $A^{r}$ is a mixed Shimura variety over $S=S^{K}(G, \mathcal{H})$. More precisely, the representation $\operatorname{Res}_{L / \mathbb{Q}} V$ of $G$ is easily seen to be of Hodge type $\{(-1,0),(0,-1)\}$ in the sense of $[\mathrm{P}$, Sect. 2.16]. The same statement is then true for the $r$-th power $\operatorname{Res}_{L / \mathbb{Q}} V^{r}$ of $\operatorname{Res}_{L / \mathbb{Q}} V$. By [P. Prop. 2.17], this allows for the construction of the unipotent extension $\left(P^{r}, \mathfrak{X}^{r}\right)$ of $(G, \mathcal{H})$ by $\operatorname{Res}_{L / \mathbb{Q}} V^{r}$. The reader wishing an explicit description of $\left(P^{r}, \mathfrak{X}^{r}\right)$ is referred to [K, Sect. 1.1], where the case $g=2$ is treated. The description from [loc. cit.] generalizes easily to arbitrary $g$. 
The pair $\left(P^{r}, \mathfrak{X}^{r}\right)$ constitute mixed Shimura data $[\mathrm{P}$, Def. 2.1]. By construction, they come endowed with a morphism $\pi^{r}:\left(P^{r}, \mathfrak{X}^{r}\right) \rightarrow(G, \mathcal{H})$ of Shimura data, identifying $(G, \mathcal{H})$ with the pure Shimura data underlying $\left(P^{r}, \mathfrak{X}^{r}\right)$. In particular, $\left(P^{r}, \mathfrak{X}^{r}\right)$ also satisfy condition $(+)$ from [W3, Sect. 5].

Now there is an open compact neat subgroup $K^{r}$ of $P^{r}\left(\mathbb{A}_{f}\right)$, whose image under $\pi^{r}$ equals $K$, and such that $A^{r}$ is identified with the mixed Shimura variety $S^{K^{r}}\left(P^{r}, \mathfrak{X}^{r}\right)[\mathrm{P}$, Sect. 3.22, Thm. 11.18 and 11.16]. Furthermore, the morphism $\pi^{r}$ of Shimura data induces a morphism $S^{K^{r}}\left(P^{r}, \mathfrak{X}^{r}\right) \rightarrow S^{K}(G, \mathcal{H})$, which is identified with the structure morphism of $A^{r}$.

In order to obtain control on the boundary motive of $A^{r}$, we fix a smooth toroidal compactification $\widetilde{A^{r}}$. It is associated to a $K^{r}$-admissible complete smooth cone decomposition $\mathfrak{S}$, i.e., a collection of subsets of

$$
\mathcal{C}\left(P^{r}, \mathfrak{X}^{r}\right) \times P^{r}\left(\mathbb{A}_{f}\right)
$$

satisfying the axioms of $\left[\mathrm{P}\right.$, Sect. 6.4]. Here, $\mathcal{C}\left(P^{r}, \mathfrak{X}^{r}\right)$ denotes the conical complex associated to $\left(P^{r}, \mathfrak{X}^{r}\right)[\mathrm{P}$, Sect. 4.24].

We refer to $[\mathrm{P}, 9.27,9.28]$ for criteria sufficient to guarantee the existence of the associated compactification $\widetilde{A^{r}}:=S^{K^{r}}\left(P^{r}, \mathfrak{X}^{r}, \mathfrak{S}\right)$. It comes equipped with a natural (finite) stratification into locally closed strata. The unique open stratum is $A^{r}$. Any stratum $\widetilde{A_{\sigma}^{r}}$ different from the generic one is associated to a rational boundary component $\left(P_{1}, \mathfrak{X}_{1}\right)$ of $\left(P^{r}, \mathfrak{X}^{r}\right)[\mathrm{P}$, Sect. 4.11] which is proper, i.e., unequal to $\left(P^{r}, \mathfrak{X}^{r}\right)$.

First, co-localization for the boundary motive [W2, Cor. 3.5] tells us that $\partial M_{g m}\left(A^{r}\right)$ is a successive extension of (shifts of) objects of the form

$$
M_{g m}\left(\widetilde{A_{\sigma}^{r}}, i_{\sigma}^{!} j_{!} \mathbb{Z}\right) \text {. }
$$

Here, $j$ denotes the open immersion of $A^{r}$ into $\widetilde{A^{r}}, i_{\sigma}$ runs through the immersions of the strata $\widetilde{A_{\sigma}^{r}}$ different from $A^{r}$ into $\widetilde{A^{r}}$, and $M_{g m}\left(\widetilde{A_{\sigma}^{r}}, i_{\sigma}^{!} j_{!} \mathbb{Z}\right)$ is the motive of $\widetilde{A_{\sigma}^{r}}$ with coefficients in $i_{\sigma}^{!} j ! \mathbb{Z}$ defined in [W2, Def. 3.1].

Next, by [W3, Thm. 6.1], there is an isomorphism

$$
M_{g m}\left(\widetilde{A_{\sigma}^{r}}, i_{\sigma}^{!} j_{!} \mathbb{Z}\right) \stackrel{\sim}{\longrightarrow} \operatorname{Hom}\left(\mathbb{Z}(\sigma), M_{g m}\left(S^{K_{1}}\left(P_{1}, \mathfrak{X}_{1}\right)\right)\right)[\operatorname{dim} \sigma] .
$$

Recall [W3, p. 971] that the group of orientations $\mathbb{Z}(\sigma)$ is (non-canonically) isomorphic to $\mathbb{Z}$, hence

$$
\operatorname{Hom}\left(\mathbb{Z}(\sigma), M_{g m}\left(S^{K_{1}}\left(P_{1}, \mathfrak{X}_{1}\right)\right)\right) \cong M_{g m}\left(S^{K_{1}}\left(P_{1}, \mathfrak{X}_{1}\right)\right) .
$$

$S^{K_{1}}\left(P_{1}, \mathfrak{X}_{1}\right)$ is a Shimura variety associated to the data $\left(P_{1}, \mathfrak{X}_{1}\right)$ and an open compact neat subgroup $K_{1}$ of $P_{1}\left(\mathbb{A}_{f}\right)$. In order to show our claim, we are thus reduced to showing that $M_{g m}\left(S^{K_{1}}\right)$ is an object of $D M D T(\mathbb{Q})_{\mathbb{Q}}$, for any Shimura variety $S^{K_{1}}=S^{K_{1}}\left(P_{1}, \mathfrak{X}_{1}\right)$ associated to a proper rational boundary component $\left(P_{1}, \mathfrak{X}_{1}\right)$ of $\left(P^{r}, \mathfrak{X}^{r}\right)$, and any open compact neat subgroup $K_{1}$ of $P_{1}\left(\mathbb{A}_{f}\right)$.

Given that $P^{r}$ is a unipotent extension of $G$, the pure Shimura data underlying $\left(P_{1}, \mathfrak{X}_{1}\right)$ coincides with the pure Shimura data underlying some 
proper rational boundary component $\left(G_{1}, \mathcal{H}_{1}\right)$ of $(G, \mathcal{H})$. By definition $[\mathrm{P}$, Sect. 4.11], the group $G_{1}$ is associated to an admissible $\mathbb{Q}$-parabolic subgroup $Q$ of $G[\mathrm{P}$, Def. 4.5]. It is not difficult to see that the inverse image under the immersion of $G$ into $\operatorname{Res}_{L / \mathbb{Q}} \mathrm{GL}_{2, L}$ induces a bijection on the sets of $\mathbb{Q}$-parabolic subgroups. Under this bijection, the group $Q$ corresponds necessarily to a group of the form $\operatorname{Res}_{L / \mathbb{Q}} B$, for some Borel subgroup $B$ of $\mathrm{GL}_{2, L}$. Equivalently, $Q$ is the stabilizer in $G$ of a subspace of $\operatorname{Res}_{L / \mathbb{Q}} V$ of the form $\operatorname{Res}_{L / \mathbb{Q}} V^{\prime}$, for a one-dimensional $L$-subspace $V^{\prime}$ of $V$. A computation analogous to the one from [P, Ex. 4.25] for $g=1$ shows that the pure Shimura data underlying $\left(G_{1}, \mathcal{H}_{1}\right)$ equal the data $\left(\mathbb{G}_{m, \mathbb{Q}}, \mathcal{H}_{0}\right)$ from $[\mathrm{P}$, Ex. 2.8]. Altogether, we see that $\left(P_{1}, \mathfrak{X}_{1}\right)$ is a unipotent extension of $\left(\mathbb{G}_{m, \mathbb{Q}}, \mathcal{H}_{0}\right)$.

We are ready to conclude. As follows directly from the definition of the canonical model (cmp. [P, Sect. 11.3, 11.4]), the pure Shimura variety $S^{\pi^{r}\left(K_{1}\right)}\left(\mathbb{G}_{m, \mathbb{Q}}, \mathcal{H}_{0}\right)$ underlying $S^{K_{1}}$ equals the spectrum of a cyclotomic field $C$ over $\mathbb{Q}$. By $\left[\mathrm{P}\right.$, Prop. 11.14], the variety $S^{K_{1}}$ is isomorphic to a power of the multiplicative group over $C$. In particular, its motive lies in $\operatorname{DMDT}(\mathbb{Q})_{\mathbb{Q}}$.

q.e.d.

Remark 4.3. As the proof shows, Theorem 4.2 admits a version "before tensoring with $\mathbb{Q}$ ". That is, the boundary motive $\partial M_{g m}\left(A^{r}\right)$ lies in the triangulated sub-category $\operatorname{DMDT}(\mathbb{Q})$ of $D M_{g m}(\mathbb{Q})$ generated by Tate twists and motives $M_{g m}(\operatorname{Spec} k)$, for Abelian finite field extensions $k$ of $\mathbb{Q}$.

In order to apply the results from Section 2, we need to analyze the Hodge structure on the $e_{\underline{r}}$-part of the boundary cohomology of $A^{r}$,

$$
\left(\partial H^{n}\left(A^{r}(\mathbb{C}), \mathbb{Q}\right) \otimes_{\mathbb{Q}} F\right)^{e_{\underline{r}}},
$$

for all integers $n$. Recall that there is a canonical isomorphism

$$
\left(\operatorname{Res}_{L / \mathbb{Q}} V\right) \otimes_{\mathbb{Q}} F \stackrel{\sim}{\longrightarrow} \bigoplus_{\sigma \in I_{L}} V_{\sigma},
$$

where we set $V_{\sigma}:=V \otimes_{L, \sigma} F$. In fact, this is an isomorphism of representations of $G$ over $F$.

Definition 4.4. Denote by $V_{\underline{r}}$ the representation

$$
V_{\underline{r}}:=\bigotimes_{\sigma \in I_{L}} \operatorname{Sym}^{r_{\sigma}} V_{\sigma}^{\vee}
$$

of $G$ over $F$.

The tensor product is over $F$, and $V_{\sigma}^{\vee}$ is the contragredient representation of $V_{\sigma}$. Recall (e.g. [W1, Thm. 2.2]) the definition of the canonical construction functor $\mu$ from the category of finite-dimensional algebraic representations of $G$ to the category of admissible graded-polarizable variations of Hodge structure on $S$. 
Proposition 4.5. There is a canonical isomorphism of Hodge structures $\left(\partial H^{n}\left(A^{r}(\mathbb{C}), \mathbb{Q}\right) \otimes_{\mathbb{Q}} F\right)^{e_{\underline{r}}} \stackrel{\sim}{\longrightarrow} \partial H^{n-r}\left(S(\mathbb{C}), \mu\left(V_{\underline{r}}\right)\right)$

for all integers $n$.

Proof. The central observation is that the functor $\mu$ maps $\operatorname{Res}_{L / \mathbb{Q}} V^{\vee}$ to the first higher direct image of $\mathbb{Q}(0)$ under the structure morphism of the Abelian variety $A[\mathrm{~W} 1$, remark following Lemma 2.5]. The rest of the argument is purely formal; cmp. [K, proof of Prop. 2.3.3] for the case $g=2$.

q.e.d.

Thus, we need to control $\partial H^{n-r}\left(S(\mathbb{C}), \mu\left(V_{r}\right)\right)$. As the reader may expect, we use the Baily-Borel compactification $\bar{S}^{*}$ of $S$. The complement of $S$ consists of finitely many cusps; the boundary cohomology of $S(\mathbb{C})$ therefore coincides with the direct sum over the cusps of the degeneration of the coefficients to the boundary of $S^{*}$.

Proof of Theorem 3.6. By assumption, there are $\tau, \sigma \in I_{L}$ such that $r_{\tau} \neq r_{\sigma}$. This implies [BrL, Lemme 2.2.8] that the boundary cohomology of $\mu\left(V_{r}\right)$ vanishes. Proposition 2.5 tells us that the Hodge theoretic realization of $\partial M_{g m}\left(A^{r}\right)^{e_{\underline{r}}}$ is zero. But $\partial M_{g m}\left(A^{r}\right)^{e_{\underline{r}}}$ is Artin-Tate (Theorem4.2 and [W5, Cor 2.6]). Given that the realization is conservative on Artin-Tate motives [W5, Cor. $3.10(\mathrm{a})$ ], this means that $\partial M_{g m}\left(A^{r}\right)^{e_{\underline{r}}}$ is itself zero.

q.e.d.

Proof of Theorem 3.5. The first claim is Theorem 4.2. Given Theorem [3.6, we may assume that all $r_{\sigma}$ are equal,

$$
r_{\sigma}=s \geq 0 \forall \sigma \in I_{L},
$$

say. Thus,

$$
V_{\underline{r}}=\bigotimes_{\sigma \in I_{L}} \operatorname{Sym}^{s} V_{\sigma}^{\vee}
$$

and $r=g \cdot s$. Note that this representation descends to $\mathbb{Q}$, and that it occurs as a direct factor of the representation $\operatorname{Sym}^{r} \operatorname{Res}_{L / \mathbb{Q}} V^{\vee}$. Fix a cusp $y$ of $S^{*}(\mathbb{C})$, and denote by $j$ the open immersion of $S$ into $S^{*}$. We need to compute the weights occurring in

$$
R^{n-r} j_{*}\left(\mu\left(V_{\underline{r}}\right)\right)_{y} .
$$

First [BrL, bottom of p. 386], the cup product

$$
R^{0} j_{*}\left(\mu\left(V_{\underline{r}}\right)\right)_{y} \otimes_{\mathbb{Q}} R^{n-r} j_{*}(\mathbb{Q}(0))_{y} \longrightarrow R^{n-r} j_{*}\left(\mu\left(V_{\underline{r}}\right)\right)_{y}
$$

is an isomorphism in degrees $0 \leq n-r \leq g-1$. Next [BrL, Thm. 1.3.4, Cor. 1.3.7], in the same range of indices, the map induced by the cup product

$$
\Lambda^{n-r} R^{1} j_{*}(\mathbb{Q}(0))_{y} \longrightarrow R^{n-r} j_{*}(\mathbb{Q}(0))_{y}
$$

is an isomorphism. We shall show: 
(1) $R^{0} j_{*}\left(\mu\left(V_{\underline{r}}\right)\right)_{y} \cong \mathbb{Q}(0)$ as Hodge structures,

(2) $R^{1} j_{*}(\mathbb{Q}(0))_{y} \cong \mathbb{Q}(0)^{g-1}$ as Hodge structures, when $g \geq 2$.

Admitting these claims for the moment, we see from (1) and (2) that the Hodge structure $R^{n-r} j_{*}\left(\mu\left(V_{\underline{r}}\right)\right)_{y}$ is pure of weight 0 for $0 \leq n-r \leq g-1$, i.e., $r \leq n \leq r+g-1$. In particular, $R^{n-r} j_{*}\left(\mu\left(V_{\underline{r}}\right)\right)_{y}$ is without weights $n-(r-1), \ldots, n+r$ whenever $n \leq r+g-1$. To deal with the complementary range of indices $n \geq r+g$, recall that the Hodge structures

$$
R^{m} j_{*}\left(\mu\left(V_{\underline{r}}\right)\right)_{y} \quad \text { and } \quad R^{2 g-1-m} j_{*}\left(\mu\left(V_{\underline{r}}\right)\right)_{y}(r+g)
$$

are dual to each other, for all integers $m$. Indeed, the representation $V_{\underline{r}}$ is pure of weight $r$ in the sense of [P, Sect. 1.11], therefore [P, Prop. 1.12] it can be $G$-equivariantly identified with its own contragredient, twisted by $\mathbb{Q}(-r)$, i.e.,

$$
\mu\left(V_{\underline{r}}\right) \cong \mu\left(V_{\underline{r}}\right)^{\vee}(-r)
$$

as variations of Hodge structures. Given the definition of the Verdier dual $\mathbb{D}$ in the category of algebraic Hodge modules [St, Prop. 2.6], we have

$$
\mu\left(V_{\underline{r}}\right) \cong \mathbb{D}_{S}\left(\mu\left(V_{\underline{r}}\right)\right)(-(r+g))[-2 g] .
$$

Denoting by $i$ the immersion of $y$ into $S^{*}$, we have

$$
i^{*} \circ R j_{*} \circ \mathbb{D}_{S}=\mathbb{D}_{y} \circ i^{!} \circ j_{!} ;
$$

furthermore, $i^{!} \circ j_{!}=i^{*} \circ R j_{*}[1]$ [St, formulae (4.3.5) and (4.4.1)]. This implies that

$$
i^{*} R j_{*} \mu\left(V_{\underline{r}}\right) \cong \mathbb{D}_{y} i^{*} R j_{*} \mu\left(V_{\underline{r}}\right)(-(r+g))[-2 g+1] .
$$

Therefore, we see that $R^{n-r} j_{*}\left(\mu\left(V_{\underline{r}}\right)\right)_{y}$ is pure of weight $2(r+g)$ whenever $g \leq n-r \leq 2 g-1$, i.e., $r+g \leq n \leq r+2 g-1$. In particular, $R^{n-r} j_{*}\left(\mu\left(V_{\underline{r}}\right)\right)_{y}$ is without weights $n-(r-1), \ldots, n+r$ in any case. Given Theorem 4.2, we thus have verified the hypotheses of Corollary 2.4.

It remains to show claims (1) and (2). We shall use the main result from $\mathrm{BuW}$ on degeneration in the Baily-Borel compactification of variations in the image of $\mu$. The cusp $y$ belongs to one of the strata associated to a rational boundary component $\left(P_{1}, \mathfrak{X}_{1}\right)$ of $(G, \mathcal{H})$, where $P_{1}$ is contained as a normal subgroup in one of the admissible $\mathbb{Q}$-parabolic subgroups $Q$ of $G[\mathrm{P}$, Sect. 4.11]. The latter being the stabilizer in $G$ of a subspace of $\operatorname{Res}_{L / \mathbb{Q}} V$ of the form $\operatorname{Res}_{L / \mathbb{Q}} V^{\prime}$, for a one-dimensional $L$-subspace $V^{\prime}$ of $V$, we see that the situation is conjugate under an element of $G(\mathbb{Q})$ to the one associated to the standard Borel subgroup. Since claims (1) and (2) are invariant under isomorphisms, we may therefore assume that we work in this setting.

It is identical to the one considered in [B], proof of Prop. 3.2] (with the same notation). Applying [BuW, Thm. 2.9], we see that

$$
R^{0} j_{*}\left(\mu\left(V_{\underline{r}}\right)\right)_{y} \cong H^{0}\left(\bar{H}_{C}, H^{0}\left(W_{1}, \operatorname{Res}_{Q}^{G} V_{\underline{r}}\right)\right) \text {, }
$$


while

$$
R^{1} j_{*}(\mathbb{Q}(0))_{y} \cong H^{0}\left(\bar{H}_{C}, H^{1}\left(W_{1}, \mathbb{Q}(0)\right)\right) \oplus H^{1}\left(\bar{H}_{C}, H^{0}\left(W_{1}, \mathbb{Q}(0)\right)\right) .
$$

Here, $W_{1}$ denotes the unipotent radical of $Q$, and $\bar{H}_{C}$ is free Abelian of rank $g-1$ (cmp. [Bl, Sect. 3.2]). Note that there is a shift by the codimension of $y$ in $S^{*}$ (which equals $g$ ) in the formula of [BuW, Thm. 2.9], due to the normalization of the inclusion of the category of variations into the derived category of algebraic Hodge modules used in [loc. cit.]. In order to evalute the first of the above expressions, one proceeds dually to [Bl, proof of Prop. 3.2], to show:

(3) $H^{0}\left(W_{1}, \operatorname{Res}_{Q}^{G} V_{\underline{r}}\right)$ is one-dimensional,

(4) the actions of $\bar{H}_{C}$ and of $P_{1} / W_{1}$ on $H^{0}\left(W_{1}, \operatorname{Res}_{Q}^{G} V_{\underline{r}}\right)$ are both trivial.

Given that the action of $P_{1} / W_{1}$ determines the Hodge structure, this shows (1). As for (2), note first that

$$
H^{0}\left(\bar{H}_{C}, H^{1}\left(W_{1}, \mathbb{Q}(0)\right)\right)=0
$$

when $g \geq 2[$ BrL, bottom of p. 386]. Hence

$$
R^{1} j_{*}(\mathbb{Q}(0))_{y} \cong H^{1}\left(\bar{H}_{C}, H^{0}\left(W_{1}, \mathbb{Q}(0)\right)\right)
$$

in this case. Given that $H^{0}\left(W_{1}, \mathbb{Q}(0)\right)=\mathbb{Q}(0)$, and that the action of $\bar{H}_{C}$ on $H^{0}\left(W_{1}, \mathbb{Q}(0)\right)$ is trivial (use claim (4) for $s=0$ ), we have indeed

$$
R^{1} j_{*}(\mathbb{Q}(0))_{y} \cong \operatorname{Hom}\left(\bar{H}_{C}, \mathbb{Q}\right)(0) \text {. }
$$

q.e.d.

\section{References}

[An] Y. André, Une introduction aux motifs, Panoramas et Synthèses 17, Soc. Math. France (2004).

[Ay] J. Ayoub, Note sur les opérations de Grothendieck et la réalisation de Betti, J. Inst. Math. Jussieu 9 (2010), 225-263.

[Be1] A.A. Beilinson, Higher regulators of modular curves, in: S.J. Bloch, R. Keith Dennis, E.M. Friedlander, M.R. Stein (eds.), Applications of Algebraic K-Theory to Algebraic Geometry and Number Theory. Proceedings of the AMS-IMS-SIAM Joint Summer Research Conference held at the University of Colorado, Boulder, Colorado, June 12-18, 1983, Contemp. Math. 55 (1986), 1-34. 
[Be2] A.A. Beilinson, Notes on absolute Hodge cohomology, in: S.J. Bloch, R. Keith Dennis, E.M. Friedlander, M.R. Stein (eds.), Applications of Algebraic K-Theory to Algebraic Geometry and Number Theory. Proceedings of the AMS-IMS-SIAM Joint Summer Research Conference held at the University of Colorado, Boulder, Colorado, June 12-18, 1983, Contemp. Math. 55 (1986), 35-68.

[Bl] D. Blottière, Les classes d'Eisenstein des variétés de HilbertBlumenthal, Int. Math. Res. Notices 2009 (2009), 3236-3263.

[Bo] M.V. Bondarko, Weight structures vs. t-structures; weight filtrations, spectral sequences, and complexes (for motives and in general), J. KTheory 6 (2010), 387-504.

[BrL] J.-L. Brylinski, J.-P. Labesse, Cohomologie d'intersection et fonctions $L$ de certaines variétés de Shimura, Ann. Scient. ENS 17 (1984), 361412.

[BuW] J.I. Burgos, J. Wildeshaus, Hodge modules on Shimura varieties and their higher direct images in the Baily-Borel compactification, Ann. Scient. ENS. 37 (2004), 363-413.

[CMi] M.A.A. de Cataldo, L. Migliorini, The Chow motive of semismall resolutions, Math. Res. Lett. 11 (2004), 151-170.

[D] P. Deligne, Formes modulaires et représentations $\ell$-adiques, Sém. Bourbaki, Exposé 355, Lect. Notes Math. 179, Springer-Verlag (1969), 139172 .

[DG] P. Deligne, A.B. Goncharov, Groupes fondamentaux motiviques de Tate mixte, Ann. Scient. ENS 38 (2005), 1-56.

[DeM] C. Deninger, J. Murre, Motivic decomposition of abelian schemes and the Fourier transform, J. reine angew. Math 422 (1991), 201-219.

[DiT] M. Dimitrov, J. Tilouine, Variétés et formes modulaires de Hilbert arithmétiques pour $\Gamma_{1}(\mathfrak{c}, \mathfrak{n})$, in A. Adolphson et al. (eds.), Geometric Aspects of Dwork Theory, de Gruyter (2004), 555-614.

[E] T. Ekedahl, On the adic formalism, in P. Cartier et al. (eds.), The Grothendieck Festschrift, Volume II, Prog. in Math. 87, BirkhäuserVerlag (1990), 197-218.

[F] E. Freitag, Hilbert modular forms, Springer-Verlag (1990).

[Gh] E. Ghate, Adjoint L-Values and Primes of Congruence for Hilbert Modular Forms, Compositio Math. 132 (2002), 243-281. 
[GHM1] B.B. Gordon, M. Hanamura, J.P. Murre, Relative Chow-Künneth projectors for modular varieties, J. reine angew. Math 558 (2003), 1-14.

[GHM2] B.B. Gordon, M. Hanamura, J.P. Murre, Absolute Chow-Künneth projectors for modular varieties, J. reine angew. Math 580 (2005), 139155.

[Ha] G. Harder, Eisenstein cohomology of arithmetic groups. The case $\mathrm{GL}_{2}$, Invent. Math. 89 (1987), 37-118.

[Hu] A. Huber, Realization of Voevodsky's motives, J. of Alg. Geom. 9 (2000), 755-799, Corrigendum, 13 (2004), 195-207.

[K] G. Kings, Higher regulators, Hilbert modular surfaces, and special values of L-functions, Duke Math. J. 92 (1998), 61-127.

[P] R. Pink, Arithmetical compactification of mixed Shimura varieties, Bonner Mathematische Schriften 209, Univ. Bonn (1990).

[R] M. Rapoport, Compactification de l'espace de modules de HilbertBlumenthal, Compositio Math. 36 (1978), 255-335.

[St] M. Saito, Mixed Hodge modules, Publ. RIMS, Kyoto Univ. 26 (1990), 221-333.

[Sp] L. Saper, $\mathcal{L}$-modules and the conjecture of Rapoport and GoreskyMacPherson, in J. Tilouine, H. Carayol, M. Harris, M.-F. Vignéras (eds.), Automorphic forms. I, Astérisque 298 (2005), 319-334.

[Sch] A.J. Scholl, Motives for modular forms, Invent. Math. 100 (1990), 419430.

[V] V. Voevodsky, Triangulated categories of motives over a field, Chapter 5 of V. Voevodsky, A. Suslin, E.M. Friedlander, Cycles, Transfers, and Motivic Homology Theories, Ann. of Math. Studies 143, Princeton Univ. Press (2000).

[W1] J. Wildeshaus, The canonical construction of mixed sheaves on mixed Shimura varieties, in: Realizations of Polylogarithms, Lect. Notes Math. 1650, Springer-Verlag (1997), 77-140.

[W2] J. Wildeshaus, The boundary motive: definition and basic properties, Compositio Math. 142 (2006), 631-656.

[W3] J. Wildeshaus, On the boundary motive of a Shimura variety, Compositio Math. 143 (2007), 959-985.

[W4] J. Wildeshaus, Chow motives without projectivity, Compositio Math. 145 (2009), 1196-1226. 
[W5] J. Wildeshaus, Notes on Artin-Tate motives, 23 pages, to appear in J.-B. Bost and J.-M. Fontaine (eds.), Autour des motifs. Ecole d'été franco-asiatique de géométrie algébrique et de théorie des nombres. Vol. II, Panoramas et Synthèses, Soc. Math. France (2011), available on arXiv.org under http://xxx.lanl.gov/abs/0811.4551

[W6] J. Wildeshaus, Pure motives, mixed motives and extensions of motives associated to singular surfaces, 39 pages, to appear in J.-B. Bost and J.-M. Fontaine (eds.), Autour des motifs. Ecole d'été franco-asiatique de géométrie algébrique et de théorie des nombres. Vol. II, Panoramas et Synthèses, Soc. Math. France (2011), available on arXiv.org under http://xxx.lanl.gov/abs/0706.4447

[W7] J. Wildeshaus, Boundary motive, relative motives and extensions of motives, 46 pages, to appear in J.-B. Bost and J.M. Fontaine (eds.), Autour des motifs. Ecole d'été franco-asiatique de géométrie algébrique et de théorie des nombres. Vol. II, Panoramas et Synthèses, Soc. Math. France (2011), available on arXiv.org under http://xxx.lanl.gov/abs/1004.1631 